\title{
Simulation Method for Complex Multivalued Curves in Injection-Locked Oscillators
}

\author{
Silvia Hernández, Mabel Pontón, Member, IEEE, and Almudena Suárez, Fellow, IEEE
}

\begin{abstract}
A new methodology is presented for the efficient harmonic-balance simulation of injection-locked oscillators with complex multi-valued and disconnected curves. It is illustrated through its application to high-order subharmonically injectionlocked oscillators. A graphical technique is applied to analyze the oscillator-phase sensitivity with respect to the input signal, required for the injection-locked operation. The intricate synchronized-solution curves are obtained with the new method, which enables a global exploration of all the coexistent periodic solutions. These solutions can belong to different curve sections, in a multi-valued response, or to disconnected synchronization curves. The method is based on the calculation of a series of phasedependent outer-tier admittance functions, which provide the oscillator response to the injection signal. Coexistent solutions are simultaneously obtained through a contour-plot intersection, without the need for continuation techniques. The method is illustrated through application to an oscillator synchronized to low-frequency sinusoidal signal by means of a nonlinear transmission line (NLTL). The analysis and design techniques have been successfully validated through comparison with independent simulations and measurements.
\end{abstract}

Index Terms - Bifurcation, oscillator, phase-noise, stability, synchronization.

\section{INTRODUCTION}

L OW phase-noise is a crucial requirement of microwave

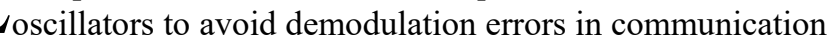
systems [1]-[4]. A comparison of state-of-the-art phase-locked loops [5]-[6] indicates that a lower voltage-controlled oscillator (VCO), followed by a frequency multiplier, enables lower phase noise than a high frequency VCO. Since efficient multipliers are limited to low multiplication factors, the authors of [5]-[6] propose the use of high-order subharmonic injection locking of an oscillator at the desired carrier frequency. The high-order subharmonic synchronization is achieved by introducing the low-frequency sinusoidal input signal into a pulse-shaping circuit [5]-[6], based on a comparator and a delay element. This provides a square pulse that switches the high frequency oscillator on and off. In [7], a similar approach is

Manuscript received December 9, 2016. This work was supported by the Spanish Ministry of Economy and Competitiveness under the research project TEC2014-60283-C3-1-R, the European Regional Development Fund (ERDF/FEDER) and Juan de la Cierva Research Program IJCI-2014-19141 and by the Parliament of Cantabria under the project Cantabria Explora 12.JP02.64069. S. Hernández, M. Pontón and A. Suárez are with the Departamento de Ingeniería de Comunicaciones, Universidad de Cantabria, Santander, 39005, Spain (e-mail: silvia.hernandez@unican.es; mabel.ponton@unican.es; almudena.suarez@unican.es). presented, using a nonlinear-transmission line (NLTL) for the pulse forming [8]-[11]. As in the case of [5]-[6], the intended application of this sub-synchronized operation is its use in low phase-noise local oscillators.

Due to the difficulties in the analysis of the high-order subharmonic injection-locked regime, approximate oscillator models are used in [5]-[6], [12]-[14], whereas the simulations in [7], [15] rely on the Poincaré-map technique [16]-[18]. This map is applied to the sequence of steady-state solutions obtained through time-domain integration of the differential algebraic equation system when varying a particular analysis parameter, such as the input power or frequency. However, the time integration may be unsuitable in some cases, for instance in the presence of distributed elements or when the circuit response involves different time scales [16]. Even when successful, it will miss coexistent stable steady-state solutions that could be relevant to its physical behavior.

This work addresses the harmonic balance (HB) analysis of high-order subharmonically injected oscillators for the first time to our knowledge. This HB analysis is well suited for circuits containing distributed elements. It is insensitive to the stability properties of the steady-state solutions, so it can provide the entire solution "path" [19]-[22] versus the analysis parameter. However, this complete portrait of the circuit response requires the use of complementary continuation techniques [23]-[25]. In fact, multivalued sections may be obtained when varying the parameter and a complementary continuation technique is needed to pass through the infiniteslope points or turning points. The subsequent application of a reliable stability analysis [26]-[29] through the entire solution curves should enable a thorough understanding of the physical evolution of the circuit solution versus the particular parameter.

Several continuation methods have been proposed for usage in combination with commercial HB [30]-[31]. However, the case of subharmonic injection-locking of a high order $N$ is particularly demanding. The circuit is in a strongly nonlinear state with respect to the input source, since the oscillation frequency must get locked to a high harmonic $N \omega_{\text {in }}$ of the input 
frequency [12]-[14]. This is different from fundamental and super-harmonic injection, in which synchronized operation bands are obtained from small-signal amplitude of the injection source [21], [31]-[32]. Obviously, the analysis will require a high number of harmonic terms and there can be a convergence failure since, besides the HB system, the solution must fulfill an additional oscillation condition. On the other hand, due to the high ratio between the oscillation frequency and injection frequency there can be synchronization at neighboring $N$ values. This may give rise to overlapped synchronization regions, which demand a global exploration capability, nonprovided by continuation procedures, which are inherently local.

The paper starts with a detailed investigation of subharmonic injection-locking, using both an analytical formulation and numerical techniques. The study will provide insight into the nonlinear mechanisms leading to this regime, as well as its particular characteristics in comparison with the more usual fundamental- and harmonic- injection-locking [12]-[14], [32]. The analysis of the phase sensitivity with respect to the input source will allow an estimation of the input power required to achieve the sub-synchronized state. The intricate synchronizedsolution curves will be obtained with a new method, based on the calculation of a series of phase-dependent admittance-type functions, constituting an outer-tier description of the oscillator response to the injection source, which is different from the undriven oscillator-admittance function proposed in [33].

The new method is applicable in combination with a commercial HB. The admittance functions are calculated with the aid of an auxiliary generator (AG), establishing the circuit phase reference [21]-[22]. One of these functions is obtained for each phase $\alpha$ of the input source, and the total phase interval can be limited to $(0,2 \pi / N)$ in most cases. For each $\alpha$, all the coexistent solutions are simultaneously obtained through a contour-plot intersection, with no need of continuation techniques. No oscillation conditions are imposed, so no optimization is required. Although the original target of the method has been the analysis of high-order subharmonically injection-locked oscillators, the method is of general application to highly nonlinear circuits exhibiting complex and disconnected solution curves.

The method has been tested in challenging prototypes from the simulation viewpoint, targeting the highest subharmonic order $N$. The package inductors of the NLTL varactor diodes (which would not be present in a MMIC realization) limit the input-frequency range with significant multiplication capabilities. In view of this fact, two different circuits have been analyzed: one suppressing these inductors (and providing the most complex curves) and the other one considering these parasitic inductors. In the first design, the oscillator operates at about $7.6 \mathrm{GHz}$ with sub-synchronization order $N=15$ and $N=16$. Results are validated through comparisons with AGbased parameter switching and with fully independent simulations based on the envelope-transient method [34]-[36]. In fact, the most reliable validation of a new analysis method would be based on its comparison with well-established simulation procedures. In this way, one can be sure that models for all the passive and active components are identical, unlike the situation encountered when comparing with measurements. As stated, with the varactor-package inductors, the harmonicgeneration capabilities of the NLTL significantly decrease, so the second oscillator design was carried out at the lower frequency $4.38 \mathrm{GHz}$. In this circuit complete models of all the linear and nonlinear components have been taken into account and the results have been compared with measurements.

The paper is organized as follows. Section II presents the general characteristics of subharmonic injection-locking. Section III describes the phase sensitivity analysis and its use for the estimation of the oscillator sub-synchronization capabilities. Section IV addresses the new methodology to trace the multi-valued sub-synchronized solution curves. Section V presents the experimental results.

\section{SUB-HARMONIC INJECTION LOCKING}

To get analytical insight into the subharmonic injectionlocking, a cubic-nonlinearity oscillator, with a parallel resonator, will be initially considered (Fig. 1). The nonlinear current is modeled as $i=a v+b v^{3}$, with $a<0$ and $b>0$, which implies a reduction of the negative conductance with the excitation amplitude. The oscillator is assumed to operate at about three times the frequency of the injection source, so $N=3$. Taking into account that the cubic nonlinearity does not generate even harmonics, and limiting the analysis to the fundamental frequency and the third harmonic term, the subsynchronized solution will fulfill the following steady-state system:

$$
\begin{aligned}
& I_{g} e^{j \alpha}=\left(Y_{L}(\omega)+a\right)\left|V_{1}\right| e^{j \phi}+\frac{3 b}{4}\left(\left|V_{1}\right|^{3}+2\left|V_{1}\right| V_{3}^{2}\right) e^{j \phi} \\
& +\frac{3 b}{4}\left|V_{1}\right|^{2} V_{3} e^{-j 2 \phi} \\
& 0=\left(Y_{L}(3 \omega)+a\right) V_{3}+\frac{b}{4}\left|V_{1}\right|^{3} e^{j 3 \phi}+\frac{3 b}{2}\left|V_{1}\right|^{2} V_{3}+\frac{3 b}{4} V_{3}^{3}
\end{aligned}
$$

where $Y_{L}$ is the linear admittance of the load network, the phase origin is taken at the third harmonic component of the node voltage, so $V_{3}=\left|V_{3}\right|, \alpha$ is the input-source phase and the phasor $V_{1}$ has been expressed as: $V_{1}=\left|V_{1}\right| \exp (j \phi)$. Splitting (1) into real and imaginary parts, one obtains:

$$
\begin{aligned}
& I_{T 1}^{r}=\left[(G+a)\left|V_{1}\right|+\frac{3}{4} b\left(\left|V_{1}\right|^{3}+2\left|V_{1}\right| V_{3}^{2}\right)\right] \cos \phi \\
& -\left(C \omega-\frac{1}{L \omega}\right)\left|V_{1}\right| \sin \phi+\frac{3}{4} b\left|V_{1}\right|^{2} V_{3} \cos 2 \phi-I_{g} \cos \alpha=0 \\
& I_{T 1}^{i}=\left[(G+a)\left|V_{1}\right|+\frac{3}{4} b\left(\left|V_{1}\right|^{3}+2\left|V_{1}\right| V_{3}^{2}\right)\right] \sin \phi \\
& +\left(C \omega-\frac{1}{L \omega}\right)\left|V_{1}\right| \cos \phi-\frac{3}{4} b\left|V_{1}\right|^{2} V_{3} \sin 2 \phi-I_{g} \sin \alpha=0 \\
& I_{T 3}^{r}=\left(G+a+\frac{3}{2} b\left|V_{1}\right|^{2}\right) V_{3}+\frac{3}{4} b V_{3}^{3}=-\frac{1}{4} b\left|V_{1}\right|^{3} \cos 3 \phi \\
& I_{T 3}^{i}=\left(C 3 \omega-\frac{1}{L 3 \omega}\right) V_{3}=-\frac{1}{4} b\left|V_{1}\right|^{3} \sin 3 \phi .
\end{aligned}
$$


The original free-running oscillation takes place about $3 \omega$ and is easily obtained from (2c) and (2d) by doing $I_{g}=0$ in (2a) and (2b), which leads to $\left|V_{1}\right|=0$. The left-hand side of (2c) differs from that of a fundamentally injection-locked oscillator at the input-source frequency $\omega_{s}=3 \omega$ in the additional term $3 b\left|V_{1}\right|^{2} / 2$, affecting $V_{3}$. Since $b>0$ this term will give rise to a reduction of the negative conductance with the magnitude $\left|V_{1}\right|$. Therefore, in injection-locked conditions there will be a reduction of the amplitude $V_{3}$ with respect to its original freerunning value [37]. On the other hand, the left-hand side of (2d) is formally identical to that of a fundamentally injection-locked oscillator at $\omega_{s}=3 \omega$. However, in the sub-synchronized case of (1), the injection amplitude is not constant but depends on the first harmonic voltage as $b\left|V_{1}\right|^{3} / 4$ [see (2c) and (2d)] and obviously requires nonlinear behavior with respect to the input source at $\omega_{s}=\omega$.

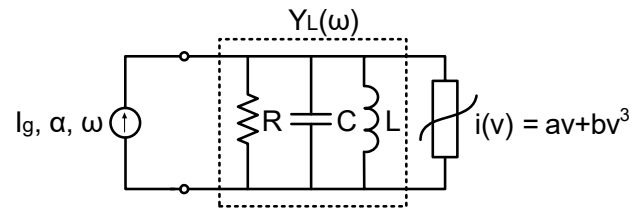

Fig. 1. Cubic-nonlinearity oscillator, loaded with a parallel resonator and injected with a current source. Element values are $C=0.5025 \mathrm{pF}, L=0.7188$ $\mathrm{nH}$ and $R=50 \mathrm{Ohm}$. The voltage-controlled current is modeled as $i(v)=-0.0432 v+0.0180 v^{3}$.

For small $I_{g}$, the magnitude of $\left|V_{1}\right|$ will be small too and the cubic function of $V_{3}$ in (2c) will provide three solutions in a certain $\omega$ interval, near the original free-running frequency. One of these solutions corresponds to small $V_{3}$, whereas the other two solutions have a higher $V_{3}$ due to the negative conductance excess $G+a$ in (2c). On the other hand, for too high $I_{g}$, only one solution will be possible. The evolution of the synchronization curves when increasing $I_{g}$, traced in terms of the node-voltage amplitude at $3 \omega$ versus the frequency $3 \omega$, is shown in Fig. 2(a). For zero input current, one point is obtained, agreeing with the free-running oscillation. In addition, there is a trivial dc solution, not represented in Fig. 2(a). For $I_{g}=40$ $\mathrm{mA}$, a closed synchronization curve is obtained and the synchronization bandwidth is limited by two infinite-slope points. One observes the amplitude reduction with respect to free-running conditions predicted by (2). Together with the closed curve, there is an open low-amplitude curve, which can be considered as an evolution of the trivial dc solution under the periodic forcing. For higher $I_{g}$, the $V_{3}$ of the two higheramplitude solutions decreases, whereas the $V_{3}$ of the loweramplitude one increases, due to the stronger forcing $b\left|V_{1}\right|^{3} / 4$. This can be seen in Fig. 2(a). For $I_{g}=45 \mathrm{~mA}$, the closed curve drops in amplitude and the open curve increases slightly. When further increasing $I_{g}$, the upper and lower solution curves merge in a single one, which exhibits turning points. See the curve obtained for $I_{g}=54 \mathrm{~mA}$. As $I_{g}$ continues to grow, the folding disappears and the resonance becomes less pronounced. See the curve obtained for $I_{g}=70 \mathrm{~mA}$. The results of time-domain simulations have been superimposed in Fig. 2(a) and lie on the stable sections of the solution curves.

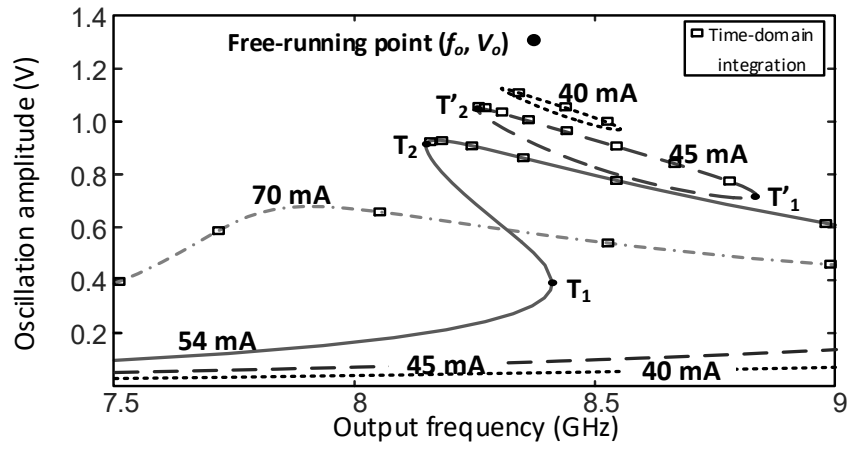

(a)

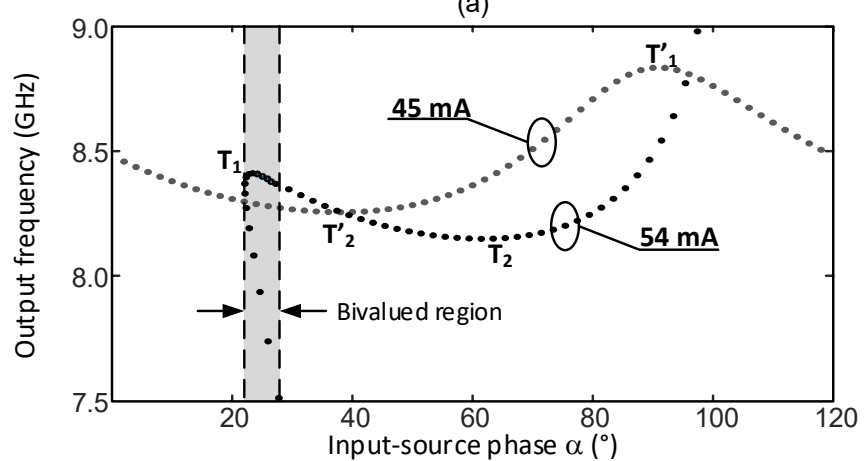

(b)

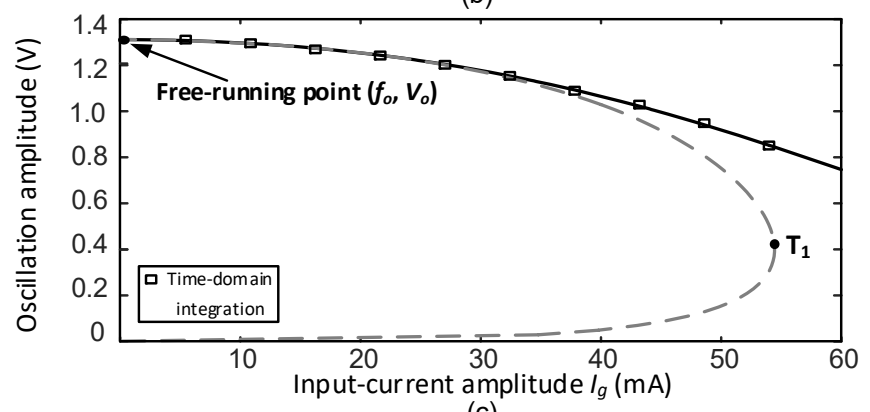

(c)

Fig. 2. Analysis of the parallel-resonance oscillator in Fig. 1. The results obtained through time-domain integration have been superimposed. (a) Synchronized-solution curves, traced in terms of the oscillation amplitude versus the input frequency, for different values of the input current $I_{g}$. (b) Variation of the input-source phase $\alpha$ versus $3 f_{\text {in }}$ for $I_{g}=45 \mathrm{~mA}$ (closed solution curve) and $I_{g}=54 \mathrm{~mA}$ (open solution curve). (c) Variation of the oscillation amplitude at $\omega_{s}=\omega_{o} / 3$, traced versus $I_{g}$.

The variations of the phase shift are analyzed next. Under small injection $b\left|V_{1}\right|^{3} / 4$ in (2c) and (2d), solutions can be obtained for the whole excursion of the sinusoidal functions $\sin (3 \phi)$ and $\cos (3 \phi)$. Thus, $V_{1}$ and $V_{3}$ will repeat with the $\phi$ period $120^{\circ}$. As a result, equations (2a) and (2b) are also periodic in $\alpha$ with the same period $120^{\circ}$. As discussed below, this periodicity will not be possible from certain injection amplitude. In Fig. 2(b) the output frequency $3 \omega_{s}$ has been represented versus $\alpha$. For $I_{g}=45 \mathrm{~mA}$, both the amplitude [Fig. 2(a)] and phase $\alpha$ exhibit two turning points versus $3 \omega_{\mathrm{s}}$, at identical values. This is because they belong to the same set of state variables evolving as a whole versus the analysis parameter $\omega_{s .}$. However, because the sinusoidal functions run through the whole interval $(-1,1)$, the curve $\alpha$ versus $\omega_{\mathrm{s}}$ is unfolded and repeats itself with the period $120^{\circ}$. 
However, from certain $I_{g}$, and due to the higher magnitude $b\left|V_{1}\right|^{3} / 4$, the system nonlinear functions cannot accommodate a complete excursion of the sinusoidal terms on the right-side of (2c) and (2d), so $\alpha$ cannot go through the full $120^{\circ}$ interval near the original resonance. In the sub-synchronized case, considered here, outside this original resonance, the phase shift $\alpha$ varies slowly versus $\omega_{s}$, due to the small $V_{3}$, enabling higher values of $|C 3 \omega-1 /(L 3 \omega)|$. In the case of a rational synchronization $m / n$, (where $m$ and $n$ are integers) considered in [38], there is no possible merging of solution curves, since the component at $m \omega_{s} / n$ can only be due to the oscillation mechanism. In that case, the synchronized solution curves keep their closed shape and the compressed phase shift necessarily evolves into a closed curve too.

Regular closed curves, having only two infinite slope points versus the input frequency, are not multi-valued with respect to the input phase, this meaning that for each $\alpha$ there is only one solution in terms of the variables $V_{1}, V_{3}, \phi, \omega$ [Fig. 2(b)]. In the intermediate $I_{g}$ interval, where the three magnitudes $I_{g}, V_{1}$ and $V_{3}$ are relatively large, more than one periodic solution can be obtained for a same $\alpha$ value. This is shown in Fig. 2(b). For $I_{g}=54 \mathrm{~mA}$, two different periodic solutions exist for each $\alpha$ value within the interval $\left(20^{\circ}, 24^{\circ}\right)$. In most cases, this is only observed after the merging of the higher and lower amplitude curves [Fig. 2(a)].

Fig. 2(c) shows the evolution of the oscillation amplitude (at $3 \omega$ ), versus the input current $I_{g}$ when setting the input frequency to exactly $\omega_{s}=\omega_{o} / 3$, where $\omega_{o}$ is the free-running frequency. Since, for $I_{g}=0$, the circuit solution agrees with the freerunning one, the curve departs from the free-running amplitude $V_{o}$. Up to $I_{g}=54 \mathrm{~mA}$, three coexistent solutions are obtained for each $I_{g}$ value. This is in correspondence with the three solutions obtained in Fig. 2(a) for low $I_{g}$. At $I_{g}=54 \mathrm{~mA}$, the curve exhibits a turning point $\left(\mathrm{T}_{1}\right)$, also observed in Fig. 2(a), at $\omega_{s}=\omega_{0} / 3$. For higher $I_{g}$, the frequency $\omega_{s}=\omega_{0} / 3$ is beyond the turning point region in Fig. 2(a), so a single solution is obtained in the representation of Fig. 2(c). Comparing Fig. 2(a) and Fig. 2(c), for relatively low input current, the synchronized solution is nearly degenerated to one point, without an actual synchronization band. This can be noted in (2c) and (2d) where such linearization should be carried out about $\left|V_{1}\right|=0$ degenerating into the original free-running point. As shown in Fig. 2(c), there is no sub-synchronized amplitude interval below $I_{g} \cong 30 \mathrm{~mA}$. In fact, for a synchronized behavior, the total current $I_{T 3}$ at $3 \omega$ (or equivalently, the total admittance function $Y_{T 3}=I_{T 3} / V_{3}$ at $3 \omega$ ) must be sensitive to phase $\alpha$. As gathered from (2d) this is only possible if $\left|V_{1}\right|^{3}$ has a sufficiently high magnitude, otherwise the circuit will always oscillate about $\omega_{o}$, so the circuit will operate in a self-oscillating mixer regime at the two fundamental frequencies $\omega_{i n}$ and $\omega_{o}$.

One major conclusion to be derived from this study is the fact that near the original resonance frequency, there is a significant compression of the $\alpha$ interval, in comparison with the regular closed curve. For intermediate values of the input amplitude, several distinct periodic solutions can be obtained for a same $\alpha$ value. This analysis of the behavior of the sub-synchronized solution curves versus the phase $\alpha$ will be helpful to understand the simulation method described in Section IV. Finally, for too large $I_{g}$, the amplitude $V_{3}$ is too small to enable multi-valued regions, and the solution curves are similar to those in ordinary resonances.

\section{Phase Sensitivity}

When simply injecting an oscillator at the free-running frequency $\omega_{o}$ with a sinusoidal signal at the frequency $\omega_{i n}$ and high ratio $\omega_{o} / \omega_{i n}$ between the two frequencies, a negligible synchronization bandwidth should be expected, even under a high input power. This is due to the limited harmonicgeneration capability of the oscillator active device(s). A multiharmonic input source (input pulse) will relax the frequencymultiplication requirements of the device nonlinearities. It is also possible to drive the circuit with a sinusoidal lowfrequency source and use a pulse-forming network to drive the oscillator circuit, such as the one in [5]-[6]. Here the pulseforming network will be implemented with a nonlinear transmission line (NLTL) [7]-[11]. NLTLs are composed by a number of inductor-varactor cells. Under a suitable design, the NLTL can give rise to a collaborative effect of the varactor diodes, in a nonlinear regime, which will lead to a high harmonic content, usually associated with soliton or shockwave formation [8]-[11].

As shown in Section II, to achieve synchronization in the order $N$, the total admittance function in $N \omega_{\text {in }}$ must be sensitive to the input-source phase $\alpha$. Here a HB technique for the analysis of the phase sensitivity with respect to the input source will be presented. This analysis enables an estimation of the sub-synchronization capabilities of a given oscillator design. For a particular $N$, the phase sensitivity is checked with the aid of a voltage auxiliary-generator (AG) [21]-[22],[31], connected in parallel at a sensitive location, such as an active-device node (Fig. 3). The AG is an independent generator [21]-[22],[31] that operates at the frequency $\omega_{A G}$ and is connected in series with an ideal bandpass filter at $\omega_{A G}$. The AG frequency should agree with the synchronized oscillation frequency, and will be expressed as $\omega_{A G}=N \omega_{i n}$. The analysis phase reference will be set at the AG voltage, so this voltage is given by $A_{A G}$. Then, the total admittance function at the AG node is the ratio $\left(Y_{A G}\right)$ between the current circulating through the AG $\left(I_{A G}\right)$ and the AG voltage $\left(A_{A G}\right)$, which is calculated with the pure HB system as an inner tier.

To check the sensitivity with respect to the input-source phase $\alpha, A_{A G}$ and $\omega_{A G}$ are set to particular values, near the free-running ones. In fact, the AG amplitude (playing the role of the oscillation) should be smaller than the free-running amplitude $V_{o}$, in consistency with the study in Section II. This will be also shown in the application example. Taking, as stated, the circuit phase origin at the AG voltage, a double sweep is performed in the input sinusoidal-signal amplitude $E_{i n}$ and phase $\alpha$, calculating the function $Y_{A G}=I_{A G} / A_{A G}$ at each sweep point. Note that it will be generally sufficient to sweep the input phase $\alpha$ 
between 0 and $2 \pi / N$ since the equations at the oscillation frequency $\left(\omega_{A G}\right)$ tend to be periodic, with the periodicity $2 \pi / N$. See (2) as an example. Due to the reduced phase interval, this sweep is not computationally expensive. To achieve synchronization the admittance function $Y_{A G}$ must be sensitive to the input phase $\alpha$.

For illustration, the phase-sensitivity analysis will be applied to the oscillator in Fig. 3, based on the transistor NE3210S01. The design is intended for operation at the output frequency 7.6 $\mathrm{GHz}$, with an output power higher than $4 \mathrm{dBm}$ When terminating the gate terminal in an inductor $L=0.85 \mathrm{nH}$, and using the capacitor values $C_{1}=2.87 \mathrm{pF}, C_{2}=0.57 \mathrm{pF}$, the circuit oscillates at $f_{o}=7.59 \mathrm{GHz}$. Nevertheless, the specified frequency $7.6 \mathrm{GHz}$ is expected to be comprised in the synchronization band, so no correction of the oscillator design has been carried out. The targeted sub-synchronization order is $N=15$. Thus, the frequency of the sinusoidal input source must be about $f_{\text {in }}=506 \mathrm{MHz}$. To achieve this high subsynchronization order, an NLTL, providing a first harmonic generation stage, will be designed. In a first stage, the varactor package inductor, which would not be present in a MMIC implementation, will not be considered. This should lead to a stronger nonlinear behavior, enabling a thorough evaluation of the capabilities of the new analysis method. The results will be validated with independent simulations. In a second stage, the package inductors will be considered. The associated low-pass filtering effects will lead to milder nonlinearities. Under this full modeling of the varactor diodes, the results will be compared with experimental measurements of the manufactured circuit.

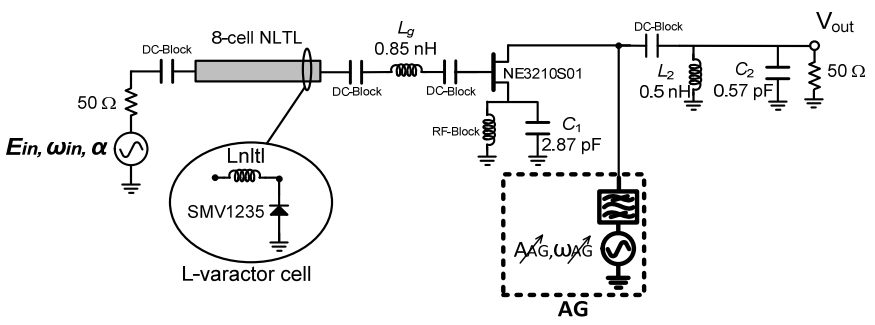

Fig. 3. First design of the subharmonically injection-locked oscillator based on the transistor NE3210S01, with ideal models of the varactor diodes, without package inductors. Results of the new analysis methodology will be compared with independent simulations.

The resulting NLTL is composed by eight $L$-varactor cells, based on the diode SMV1235 and the inductor $L=2.2 \mathrm{nH}$. When including the NLTL in the oscillator input network, the freerunning frequency is $f_{o}=7.58 \mathrm{GHz}$, still expected to be comprised in the sub-synchronization band, so no correction of the free-running oscillator design has been performed. The diagram in Fig. 4(a) shows the variation of the real and imaginary part of $Y_{A G}$ when setting $A_{A G}$ and $f_{A G}$ to the freerunning values: $A_{A G}=A_{o}=1.55 \mathrm{~V}$ and $f_{A G}=7.58 \mathrm{GHz}$ and performing a double sweep in $E_{\text {in }}$ and $\alpha$. When increasing $E_{\text {in }}$ from zero, the total admittance departs from a zero value, since the circuit fulfills the free-running oscillation conditions for zero input amplitude $\left(E_{\text {in }}=0 \mathrm{~V}\right)$. For low $E_{\text {in }}$, there is little sensitivity to the phase $\alpha$, and the admittance curves are nearly overlapped. As the amplitude increases, the real part of $Y_{A G}$ increases too and the imaginary part does not cross through zero, so the oscillation amplitude must be smaller than in freerunning regime, in agreement with the analytical study in Section II. Note that in order to get a synchronized solution, besides the sensitivity with respect to the input phase, the oscillation condition must be fulfilled, given by the zero value of the real and imaginary parts of the AG current-to-voltage ratio $Y_{A G}^{r}=0, Y_{A G}^{i}=0$, which agrees with the total admittance function at the analysis node.

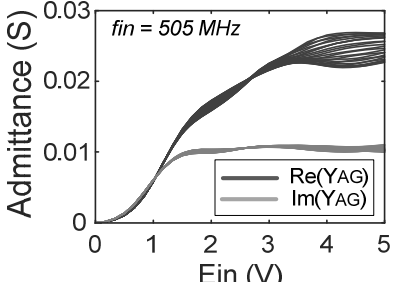

(a)

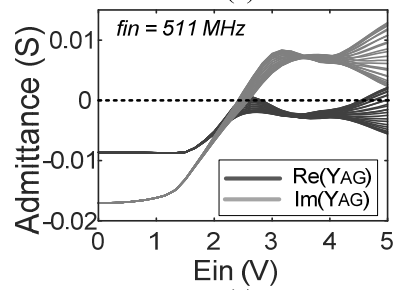

(c)

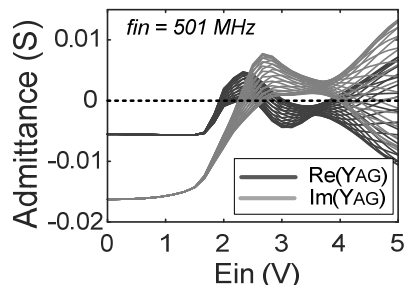

(b)

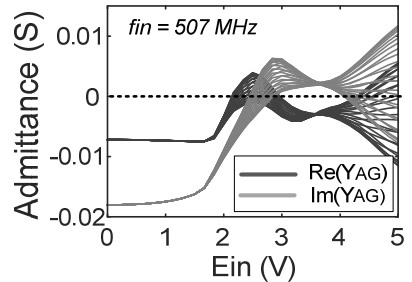

(d)
Fig. 4. Simulation of the phase-sensitivity analysis, applied to the circuit at 7.6 $\mathrm{GHz}$ in Fig. 3. (a) Variation of the admittance function $Y_{A G}$ versus $E_{\text {in }}$, for a constant AG amplitude $A_{A G}=1.55 \mathrm{~V}$ and frequency $f_{\text {in }}=505 \mathrm{MHz}$. (b) Lower frequency limit of the synchronization band, corresponding to $f_{\text {in }}=501 \mathrm{MHz}$ and an oscillation amplitude $A_{A G}=0.4 \mathrm{~V}$. (c) Higher frequency limit of the synchronization band, corresponding to $f_{\text {in }}=511 \mathrm{MHz}$ and an oscillation amplitude $A_{A G}=0.69 \mathrm{~V}$. (d) Analysis inside the synchronization band, for $f_{\text {in }}=$ $507 \mathrm{MHz}$ and $A_{A G}=0.39 \mathrm{~V}$.

In the diagrams of Fig. 4(b) to Fig. 4(d), $A_{A G}$ values smaller than the free-running amplitude and various input frequencies $f_{\text {in }}$ have been considered, with the AG frequency fulfilling $f_{\mathrm{AG}}=15 f_{\text {in }}$. For completeness, the analysis has been carried out in a large $E_{i n}$ range. In all cases, the phase sensitivity increases with $E_{\text {in }}$, then decreases (giving rise to a narrowing of the curve family) and increases again. More explicitly, there is a first input-amplitude region with high phase sensitivity, about $2 \mathrm{~V}$, and a second input-amplitude region with high-sensitivity between $4.5 \mathrm{~V}$ and $5 \mathrm{~V}$. The interesting zone for our design is the first one, since we aim at obtaining subharmonic injection locking with the lowest input power. The implications of the two zones on the solution curves versus the input power will be shown in Section IV, devoted to the accurate calculation of the steady-state solutions.

Note that the fulfillment of the steady-state conditions requires the crossing of both $Y_{A G}^{r}$ and $Y_{A G}^{i}$ through zero, which is possible for input amplitudes of about $E_{i n}=2.5 \mathrm{~V}$. This is consistent with the fact that the input source must lead the NLTL into a regime capable of generating a sufficiently high harmonic content. In Fig. 4(b), the real part is tangent to zero from above, whereas in Fig. 4(c), it is tangent to zero from 
below, which are the two limit situations for the fulfillment of the oscillation conditions. In view of Fig. 4, synchronization at constant $E_{i n}=2.5 \mathrm{~V}$ is possible for input frequencies comprised between $f_{\text {in }}=501 \mathrm{MHz}$ and $f_{\text {in }}=511 \mathrm{MHz}$. As will be shown, there is consistency between the synchronization band predicted for $E_{i n}=2.5 \mathrm{~V}$ through the phase-sensitivity analysis of Fig. 4 and the steady-state simulations in Section IV. The accuracy limitation in the analysis of Fig. 4 is due to the manual setting of the AG amplitude $A_{A G}$.

The graphical technique illustrated in Fig. 4 is computationally very cheap and efficiently combines AG method [21]-[22],[31] to obtain the steady-state synchronized solution. To calculate the synchronized solution curves versus $\omega_{i n}$, using the method described in [21]-[22],[31], one should solve the following complex equation:

$$
Y_{A G}\left(A_{A G}, \omega_{i n}, \alpha\right)=0 \text {. }
$$

Regular closed curves can be traced by simply sweeping $\alpha$ from 0 to $2 \pi / N$. This is because they are not multi-valued in the phase variable, as shown in Fig. 2(c). In contrast, multi-valued curves in $\alpha$, usually obtained for intermediate values of input amplitude, cannot be traced through this direct phase shift [Fig. 2(c)]. When using the AG, the curves multi-valued in $\alpha$, can be traced by applying parameter switching between $A_{A G}, \omega_{i n}, \alpha$ [21]-[22],[29], that is, choosing the variable with the fastest increment as the sweep parameter. In commercial HB, this can only be done manually and without any intuition on the curve shape. The particular parameter is swept until convergence fails. Then, another parameter in the set $A_{A G}, \omega_{i n}, \alpha$ is taken, restarting the simulation under a different set-up. As will be shown in Section IV, this continuation technique may be unable to provide the complete solution curves of high-order subsynchronized oscillators. This is due to the intricate solution paths resulting from the strongly nonlinear behavior with respect to the input source, in the presence of the pulse-forming network, which may include disconnected curves. The latter situation would be problematic even in in-house software. The analysis method presented in the next section is able to cope with this geometrical complexity.

\section{COnTOUR-InTERSection Method}

\section{A. Description of the analysis methodology}

The recent work [33] proposes a new technique for the efficient simulation of fundamentally injection-locked oscillators, in which neither a parameter switching nor optimization is required. The method is based on the calculation of the nonlinear admittance function that models the undriven oscillator response. This function is used at a later stage to obtain the synchronized solution when an input generator at the fundamental frequency is connected to the circuit. In a first stage, the nonlinear admittance is calculated in the absence of this input source, performing a double sweep in the AG amplitude and frequency $\left(A_{A G}, \omega_{A G}\right)$. In a second stage, the circuit is analyzed in the presence of the injection source, using the outer-tier admittance function and, in general, the Norton equivalent of the input network. This second analysis stage is enabled by the fact that the input source and the oscillation are at the same frequency $\omega_{A G}=\omega_{i n}$ in the case of a fundamentally injection-locked oscillator.

The situation is more complex in the case of subharmonically or super-harmonically injection-locked oscillators since the input signal and the oscillation are at different harmonic frequencies. This prevents the direct use of the outer-tier admittance function, modeling the sole oscillator circuit. In fact, for a direct extension of the work [33] to the analysis of a subharmonically injection-locked oscillator, one would need two different outer-tier admittance functions, one at $\omega_{A G}=N \omega_{i n}$, equated to zero, and the other at $\omega_{i n}$, which should be combined with the Norton equivalent of the input network. This implementation would be cumbersome since two complex equations and four state variables would be needed: the input frequency $\omega_{i n}$, the amplitude values at the two harmonic components $\omega_{i n}$ and $\omega_{A G}=N \omega_{i n}$ at the observation node, and the phase shift between these two harmonic components. Instead, a different method is proposed here, which takes advantage of the possibility to limit the analyzed interval of the input phases $\alpha$ to $(0,2 \pi / N)$. For relatively large $N$ values, this interval is limited to a few degrees.

As shown in Section II and III, the existence, or not, of multiple solutions for a given phase $\alpha$ depends on the inputsource amplitude. Under a given constant input-amplitude, and for each $\alpha$, each possible solution will fulfill:

$$
Y_{A G}^{\alpha}\left(A_{A G}, \omega_{A G}\right)=0
$$

where the super-index indicates that the admittance function is calculated for a particular $\alpha$, with the pure HB system, with as many harmonic components as desired, as an inner tier. The equation (3) is solved separately from the commercial HB software. In fact, this HB software is used to obtain the functions $Y_{A G}^{\alpha}$, instead of the steady-state solutions. The functions $Y_{A G}^{\alpha}$ are calculated by using a voltage AG (Fig. 3) and performing a double sweep in $A_{A G}, \omega_{A G}$ for each $\alpha$ value. At each step of the double sweep the admittance function $Y_{A G}^{\alpha}$ is calculated as the ratio between the AG current and voltage, with the current usually entering the circuit, to detect the negative conductance excess (Fig. 3). Advantage will be taken from the continuation inherent in the standard HB sweep, which uses the solution for point $n$ as an initial assumption for point $n+1$. For each $\omega_{A G}$, the amplitude $A_{A G}$ is swept from small signal to a sufficiently large value, covering the expected amplitude range, usually limited to a few volts. The number of points considered in the three sweeps (in $\alpha, A_{A G}$ and $\omega_{A G}$ ) are $N, M, P$, respectively.

In an external in-house software, two surfaces are then created for each $\alpha$, corresponding to the real and imaginary parts of $Y_{A G}^{\alpha}$. These surfaces are obtained using a 2D meshgrid, which requires the number of sweep points in $A_{A G}$ and $\omega_{A G}$ ( $M$ and $P$ ) provided by the function "sweep_size $\left(Y_{A G}\right)$ " in ADS [39]. For each $\alpha$ in the sweep, the two surfaces created independently are: 


$$
\begin{aligned}
& Y_{A G, \text { mesh }}^{\alpha, r}\left(A_{A G, m}, \omega_{A G, p}\right) \\
& Y_{A G, \text { mesh }}^{\alpha, i}\left(A_{A G, m}, \omega_{A G, p}\right)
\end{aligned}
$$

where the superindexes $r$ and $i$ indicate real and imaginary parts and $\left(A_{A G, m}, \omega_{A G, p}\right)$ represents each point of the 2D meshgrid, containing $M \times P$ points. To solve the complex equation (3), it will be necessary to interpolate the data. This interpolation is carried out using two bi-cubic polynomials in $\left(A_{A G \text {,int }}, \omega_{A G \text {,int }}\right)$, constructed as:

$$
\begin{aligned}
Y_{A G, \text { int }}^{\alpha, r} & =\sum_{l=1}^{3} \sum_{k=1}^{3} a_{m, p}^{k, l}\left(A_{A G, \text { int }}-A_{A G, m}\right)^{k}\left(\omega_{A G, \text { int }}-\omega_{A G, p}\right)^{l} \\
Y_{A G, \text { int }}^{\alpha, i} & =\sum_{l=1}^{3} \sum_{k=1}^{3} b_{m, p}^{k, l}\left(A_{A G, \text { int }}-A_{A G, m}\right)^{k}\left(\omega_{A G, \text { int }}-\omega_{A G, p}\right)^{l} .
\end{aligned}
$$

The coefficients $a_{m, p}^{k, l}$ and $b_{m, p}^{k, l}$ are calculated so as to ensure that the functions $Y_{A G \text {,int }}^{\alpha, r}$ and $Y_{A G \text {,int }}^{\alpha, i}$ are continuous and so are their partial derivatives up to second order in each of the two variables $\left(A_{A G, \text { int }}, \omega_{A G, \text { int }}\right)$ (fourth order in total). Not-a-knot end conditions [40] are imposed, this meaning that at points with coordinates $A_{A G, 2}$ and $A_{A G, M-1}$ or $\omega_{A G, 2}$ and $\omega_{A G, M-1}$, partial derivatives are continuous up to third order. The Matlab function "interp2" [40] has been used for this interpolation. This provides the surfaces:

$$
\begin{aligned}
& Y_{A G, \text { int }}^{\alpha, r}\left(A_{A G, \text { int }}, \omega_{A G, \text { int }}\right) \\
& Y_{A G, \text { int }}^{\alpha, i}\left(A_{A G, \text { int }}, \omega_{A G, \text { int }}\right) .
\end{aligned}
$$

To illustrate the method, in Fig. 5, the subharmonic order $N=15, E_{\text {in }}=2.8 \mathrm{~V}$ and input phase $\alpha=10^{\circ}$ have been considered. Fig. 5(a) shows the two interpolated surfaces, corresponding to the real and imaginary parts of $Y_{A G}^{\alpha}$.

The intersection of the surface (5a) with the plane $\operatorname{Re}\left[Y_{A G \text {,int }}^{\alpha}\right]=0$ will give rise to one or more level curves. The same is true for the intersection of surface (5b) with the plane $\operatorname{Im}\left[Y_{A G, \text { int }}^{\alpha}\right]=0$. These level curves or contours, respectively denoted as $C_{r}^{\alpha}$ and $C_{i}^{\alpha}$, are calculated setting the level of each surface in (7) to a very small value. The value $Y_{A G, \text { int }}^{\alpha, r}=Y_{A G \text {,int }}^{\alpha, i}=10^{-12}$ has been found to provide very accurate results. Thus, the zero-level curves or contours for $Y_{A G, \text { int }}^{\alpha, r}=10^{-12}$ and $Y_{A G, \text { int }}^{\alpha, i}=10^{-12}$ are calculated as:

$$
\begin{aligned}
& C_{r}^{\alpha}=\operatorname{contour}\left(A_{A G, \text { int }}, \omega_{A G, \text { int }} \mid Y_{A G, \text { int }}^{\alpha, r}=10^{-12}\right) \\
& C_{i}^{\alpha}=\operatorname{contour}\left(A_{A G, \text { int }}, \omega_{A G, \text { int }} \mid Y_{A G, \text { int }}^{\alpha, i}=10^{-12}\right) .
\end{aligned}
$$

In our practical calculation the "contour" function in Matlab [40] has been used. The contours corresponding to $E_{\text {in }}=2.8 \mathrm{~V}$ and $\alpha=10^{\circ}$ are shown at the bottom of Fig. 5(a) and in Fig. 5(b). Once the two contours have been obtained, the synchronizedsolution points are directly given by the intersections of these contours. They have been calculated using the Matlab function "polyxpoly" [40], which finds the intersection points of two lines or polygon edges in a planar, Cartersian system [40]. It returns two vectors containing the $A_{A G \text {,int }}$ and $\omega_{A G \text {,int }}$ values of each point at which the first contour intersects the second. Thus, the solution points corresponding to the particular phase value $\alpha$ are given by:

$$
\left(A_{A G . s}^{\alpha}, \omega_{A G . s}^{\alpha}\right)=C_{r}^{\alpha}\left(A_{A G, \text { int }}, \omega_{A G, \text { int }}\right) \cap C_{i}^{\alpha}\left(A_{A G, \text { int }}, \omega_{A G, \text { int }}\right)
$$

where the subindex $s$ stands for sub-synchronized solution. As stated, when obtaining the intersection of the surface with the plane $\operatorname{Re}\left[Y_{A G \text {,int }}^{\alpha}\right]=0$ or $\operatorname{Im}\left[Y_{A G, \text { int }}^{\alpha}\right]=0$, this contour may not be a single continuous level curve but a collection of them. Each of these level curves from the contour of the real part must be checked with every level curve of the collection from the contour of the imaginary part in order to avoid missing any solution point. Solutions can be traced in terms of the $P_{\text {out }}$, by simply exporting the voltage amplitude at the final $50 \mathrm{Ohm}$ load in the three-variable sweep.

In the particular case of $\alpha=10^{\circ}$, the contour intersection provides the five distinct solution points indicated with circles in Fig. 5(b). The whole synchronized-solution curve would be obtained by assembling all the intersection points resulting from the variation of $\alpha$, as shown in Fig. 5(c), where 24 values of $\alpha$ have been considered. Note that the original phase sweep considers equally-spaced phase points, though the phase value is not uniformly distributed through the synchronized solution curves. Thus, after this initial analysis, one may carry out a second one with a finer phase sweep in the regions with a lower density of solution points. One should remark that no matter the number of phase steps considered, all the points obtained from the contour intersection $C_{r}^{\alpha} \cap C_{i}^{\alpha}$ are valid solutions, since no continuation is used. Therefore, a coarse sweep would allow a global exploration of the circuit coexistent solutions, which may be helpful even in in-house HB software. Finally, in Fig. 5(d) the phase shift $\alpha$ has been represented versus $f_{\text {in }}$. In agreement with the discussion in Section II, it exhibits the same turning points as the rest of state variables, i.e., the oscillation amplitude at $15 f_{\text {in }}$ in Fig. 5(c). Due to the inherent multiplication by $N=15$, the phase variations are limited to the interval $\left(0^{\circ}\right.$, $24^{\circ}$ ).

Fig. 6 shows one sub-synchronized steady-state solution, corresponding to $f_{\text {in }}=507 \mathrm{MHz}$. Fig. 6(a) presents the pulsed voltage waveform at the NLTL output. Fig. 6(b) presents the corresponding spectrum in $\mathrm{dBV}$. We have used $\mathrm{dBV}$ for voltage measurements since the termination impedance corresponds to the complex and amplitude-dependent input impedance of the transistor-based sub-circuit. The combined multiplication action, due to the NLTL and to the transistor device in an oscillatory state can be noted. Without the latter effect, it would not be possible to sub-synchronize the oscillator at the high order $N=15$. However, the NLTL and oscillator are coupled through their input/output impedances, so they form a single oscillator circuit, sub-synchronized at the order $N$. Due to this coupling, the order $N$ will not necessarily be the result of combining the two independent multiplication orders, associated with the NLTL and the oscillatory sub-circuit. 


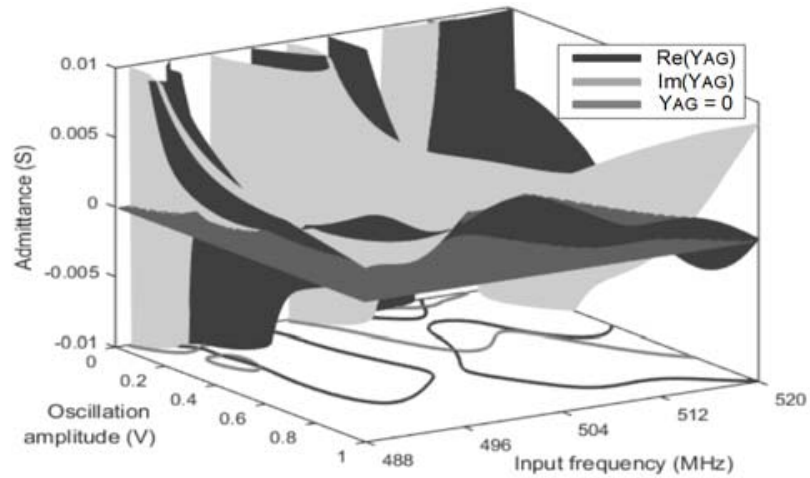

(a)

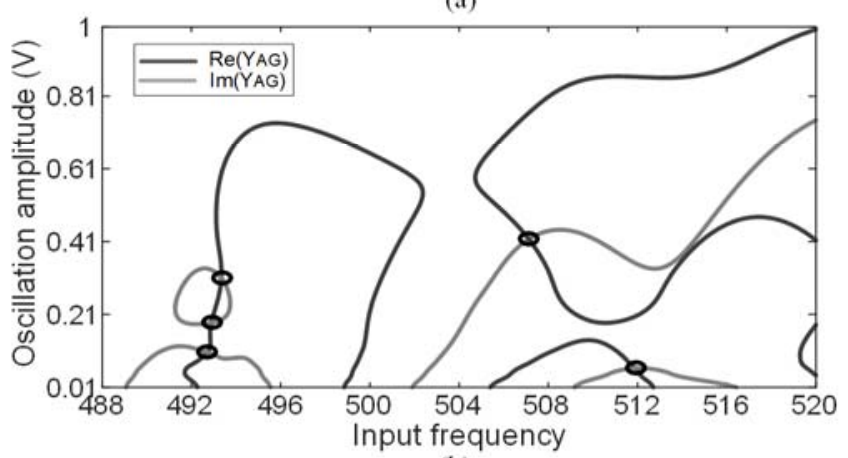

(b)

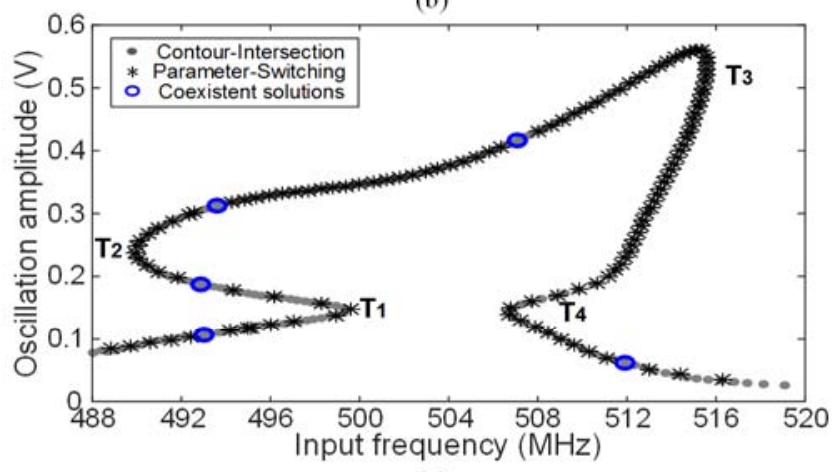

(c)

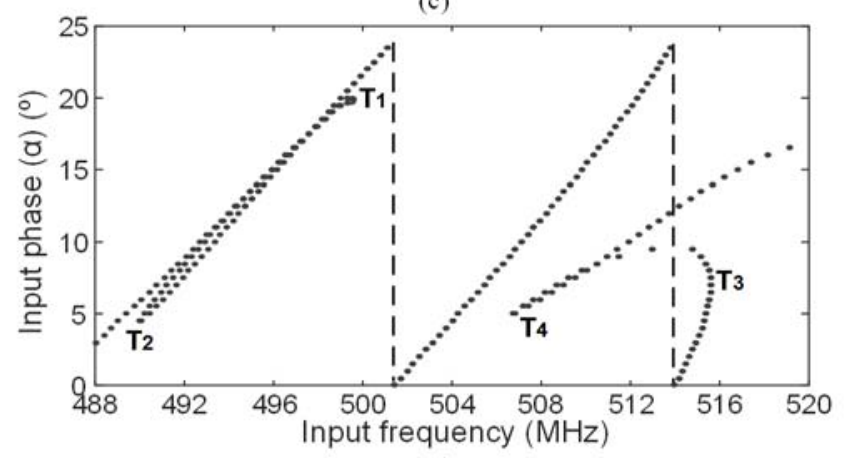

(d)

Fig. 5. Method based on the calculation of the contour-intersections, applied to the circuit at $7.6 \mathrm{GHz}$ in Fig. 3. The input amplitude is $E_{\text {in }}=2.8 \mathrm{~V}$ and the subsynchronization order is $N=15$. (a) Surfaces $\operatorname{Re}\left(Y_{A G}\right)$ and $\operatorname{Im}\left(Y_{A G}\right)$ represented versus $\left(A_{A G}, \omega_{A G}\right)$ for the particular input-phase $\alpha=10^{\circ}$. (b) Contours resulting from the intersections of the two surfaces with the planes $\operatorname{Re}\left(Y_{A G}\right)=0$ and $\operatorname{Im}\left(Y_{A G}\right)=0$. The solutions coexisting for $\alpha=10^{\circ}$ correspond to the intersections of these contours. (c) Solution curve obtained through the systematic application of this procedure for a sequence of $\alpha$ values in the interval $(0$, $360^{\circ} / N=24^{\circ}$ ). The curve obtained through AG parameter switching is superimposed for validation. The five coexistent solutions obtained in (b) for $\alpha=10^{\circ}$ are indicated with circles. (d) Variation of the phase shift $\alpha$ versus the input frequency $f_{i n}$.
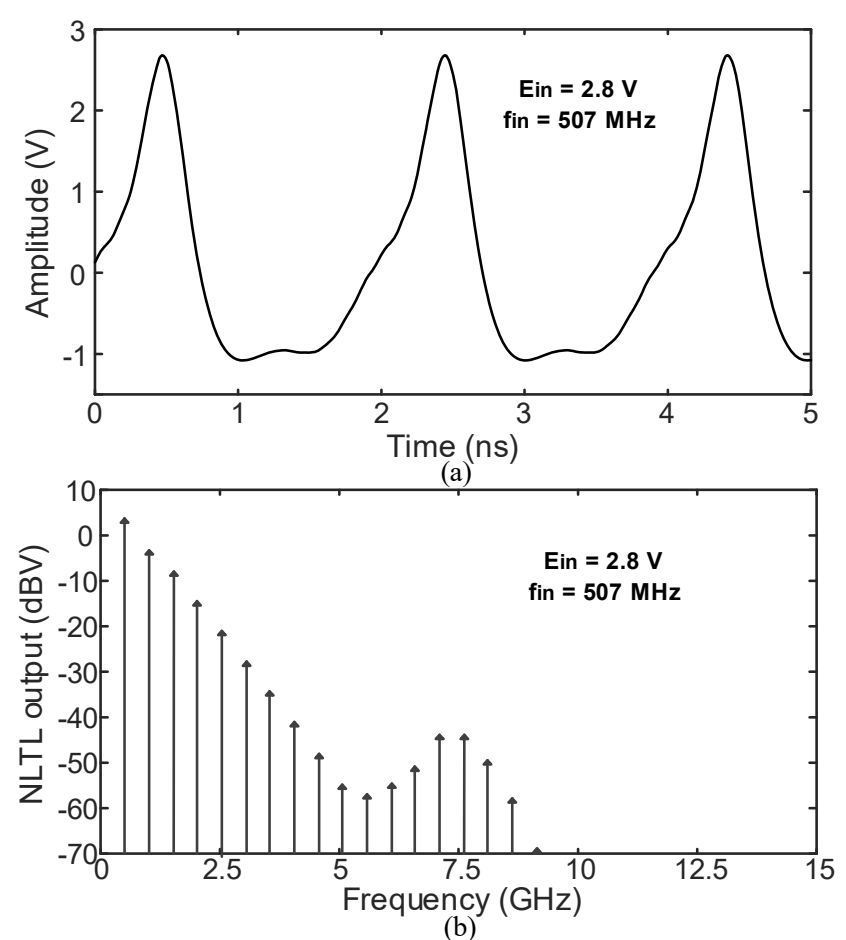

Fig. 6. Circuit at 7.6 GHz in Fig. 3. NLTL output within the oscillator circuit for input amplitude $E_{\text {in }}=2.8 \mathrm{~V}$ and frequency $f_{\text {in }}=507 \mathrm{MHz}$. (a) Pulsed waveform. (b) Spectrum.

Fig. 7(a) shows the family of synchronized solution curves obtained when increasing the input-source amplitude $E_{i n}$. The evolution is qualitatively similar to the one observed in Fig. 2(a). For $E_{\text {in }}=0$, a discrete solution point is obtained, corresponding to the free-running oscillation. Coexisting with this point, there is a trivial de solution. For the lower $E_{\text {in }}$ values $(2.5 \mathrm{~V}$ and $2.65 \mathrm{~V})$, a closed synchronization curve arises below the free-running point, which coexists with an open and lowamplitude one. The closed curve decreases in amplitude with $E_{i n}$, whereas the open curve increases, so they merge at a certain $E_{i n}$ value, only detectable as a co-dimension two bifurcation [17]. For $E_{\text {in }}=2.8 \mathrm{~V}$ the merging point has been surpassed, and a single open solution curve is obtained. For $E_{i n}=2.95 \mathrm{~V}$, the method detects two solution curves. The open curve corresponding to the subharmonic order $N=15$. The closed curve on the left side corresponds to a synchronization at the order $N=16$, since the spectral line with the highest output power is $16 \omega_{i n}$. Despite this change of order, the analysis based on the phase-dependent admittance functions was able to detect this neighboring lower frequency synchronization interval with no need to change the integer $N$ in the AG frequency, which was maintained at $\omega_{A G}=15 \omega_{i n}$. For previous $E_{i n}$ values, the curves of the $N=16$ family are too distant in frequency, so they do not appear in Fig. 7.

The entire solution curves, including the closed ones, have been obtained with the new method. Not even the closed curves could be completed with either phase sweep or parameter switching, due to an extremely high sensitivity to the phase variable in some curve sections. It is relevant to note that, as stated, for $E_{i n}=2.95 \mathrm{~V}$ the solution is composed by two 
disconnected curves. The contour intersection providing the six solutions coexisting for $\alpha=2^{\circ}$ are shown in Fig. 7(b). These solutions are indicated with circles in Fig. 7(a). Note that they belong to the two disconnected curves resulting from $E_{\text {in }}=2.95$ $\mathrm{V}$.

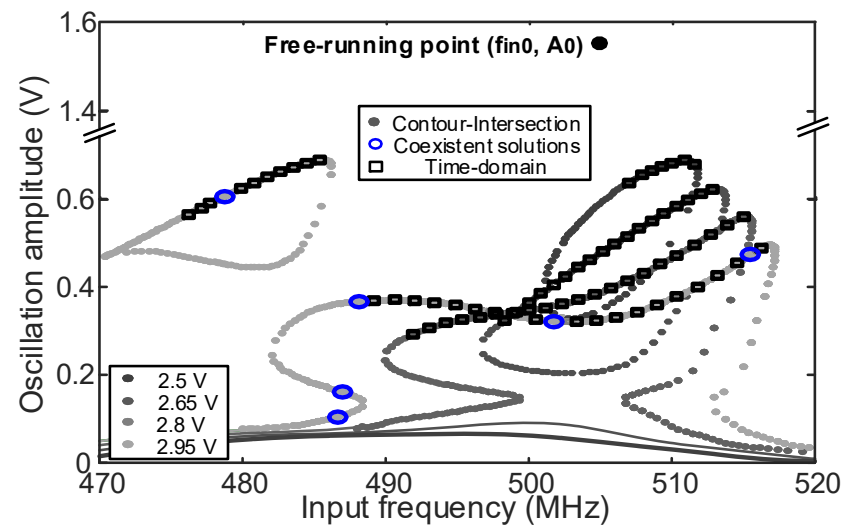

(a)

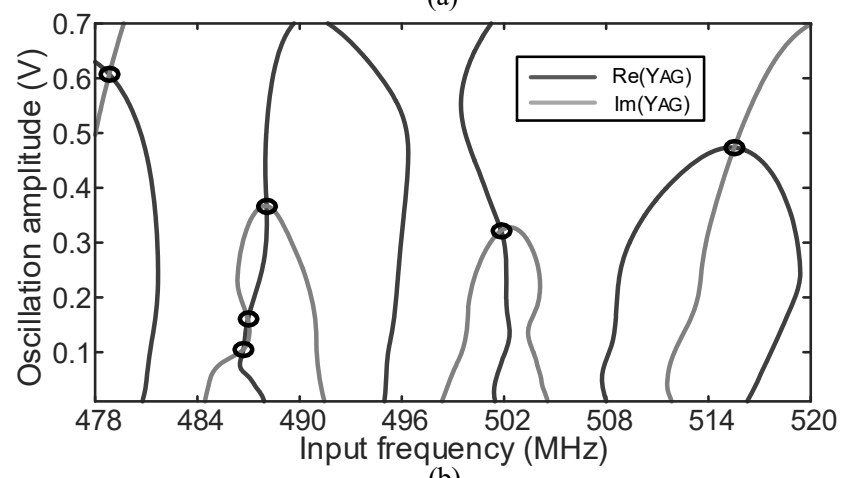

(b)

Fig. 7. Simulations of the circuit at $7.6 \mathrm{GHz}$ in Fig. 3. (a) Synchronized-solution curves versus the $f_{\text {in }}$ for different input amplitudes at the ratio $N=15$. Stable and unstable sections are indicated. The free-running solution (not represented) is given by $A_{o}=1.55 \mathrm{~V}$ and $f_{\text {in }}=505 \mathrm{MHz}$. (b) Contours resulting from the intersections of the two surfaces with the planes $\operatorname{Re}\left(Y_{A G}\right)=0$ and $\operatorname{Im}\left(Y_{A G}\right)=0$ for $E_{i n}=2.95 \mathrm{~V}$ and $\alpha=2^{\circ}$. The six coexisting solutions are indicated with circles in (a) and belong to different sections of the two disconnected curves. The results obtained through envelope-domain simulations have been superimposed in (a).

Fig. 8 shows the evolution of the amplitude at the harmonic $N=15$ traced versus the input amplitude $E_{i n}$, at the constant input frequency $f_{\text {in }}=505 \mathrm{MHz}$. The resulting curve is consistent with the decrease plus increase of the phase sensitivity with respect to the input amplitude, detected in the analysis of Fig. 4. There is one maximum at $E_{i n}=2 \mathrm{~V}$ and a positive slope section, starting from $3.75 \mathrm{~V}$. On the other hand, the multivalued nature of this curve for the lower $E_{i n}$ interval is in agreement with the initial coexistence of a closed curve plus a low amplitude curve in Fig. 7, later evolving to a single curve. The behavior is similar to the one obtained in Fig. 2(c). However, the input frequency here is different from the freerunning one, so the turning point $\mathrm{T}_{1}$ does not reach $E_{\text {in }}=0 \mathrm{~V}$.

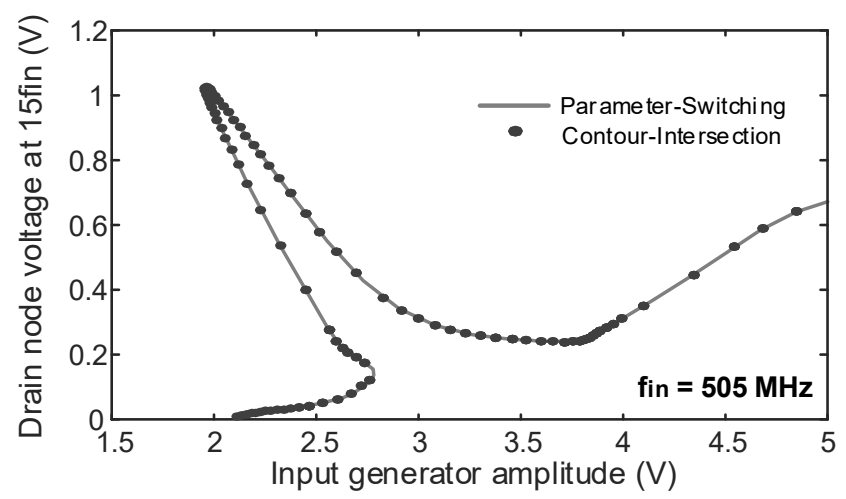

Fig. 8. Circuit at $7.6 \mathrm{GHz}$ in Fig. 3. Evolution of the amplitude at the harmonic $N=15$ traced versus the input amplitude $E_{i n}$ at the constant input frequency $f_{\text {in }}$ $=505 \mathrm{MHz}$. The results of the contour-plot intersection are compared with those obtained through AG parameter switching.

\section{B. Comparison with independent simulation techniques}

Regarding the validation of the new method with independent simulation techniques, the multi-valued curve in Fig. 5(c) agrees with the one obtained through parameter switching, which has been superimposed in Fig. 5(c). One should point out that tracing the curve in Fig. 5(c) with parameter switching has involved three manual parameter switches. In the flat region of the curve, $f_{i n}$ is swept until convergence failure. In turn, the left and right sides of the curve are obtained by sweeping $A_{A G}$ downwards. The full solution path has been composed by joining the three curve sections. Nevertheless, this procedure could not be applied in other cases reported in the next sub-section, since no convergence was found in wide regions of the solution curves, despite changing the sweep parameter. This is attributed to the strong nonlinearity of the circuit containing a first pulse-forming stage, as well as the need for a high number of harmonic terms

The purpose has also been to validate the new method with fully independent simulations, not relying on the use of any AGs. However, the time-domain simulations diverged with all the integration algorithms provided by the commercial software. Instead, the results have been validated with the envelope-transient method [34]-[36]. However, this method requires a good initial guess, otherwise it converges towards a non-oscillatory solution. The initial guess will be given by a synchronized solution point obtained with the $\mathrm{AG}$ at a particular input frequency. The AG is connected to the circuit at the initial time only, which is implemented with a time-dependent resistor, in series with AG. This resistor has a zero value at the initial time and infinite (i.e., $10^{18}$ ) afterwards [36]. The results of the first analysis point, at the frequency $\omega_{i n, 1}$ are stored in a file in the commercial HB software. This file is used as an initial guess for the next point of the sweep. Through the entire sweep, the final solution obtained at $\omega_{i n, k}$ is used as an initial guess for the next value $\omega_{i n, k+1}$ and overwritten with the solution obtained at $\omega_{i n, k+1}$. No AG is used in this procedure (except for the initial guess at $\left.\omega_{i n, 1}\right)$, so the curve obtained in this way [Fig. 7(a)] constitutes a fully independent simulation of the synchronized solution curve. In Fig. 7(a), the magnitude to the harmonic 
component $N f_{i n}$, once the steady-state is reached, has been traced versus $f_{\text {in }}$ for all the $E_{\text {in }}$ values considered. As can be seen, the curve sections obtained with envelope transient fully overlap with the curves resulting from the contour-intersection method, which validates the accuracy of the method. However, the envelope transient fails to converge near the turning points due to an ill-conditioning of the Jacobian matrix. It is also unable to pass through these turning points and provide solutions coexisting for the same frequency values.

Unlike other methodologies, the neighborhood of a turning point has no impact on the contour-intersection method. This is explained in the following. The case of the ordinary harmonicbalance (HB) system, without any auxiliary generator, will be considered first. The Jacobian matrix of this system becomes singular at any parameter value corresponding to a turning point, since at this point the solution exhibits an infinite slope versus the parameter. However, the introduction of an auxiliary generator into the circuit suppresses the system singularity. As stated in [19], the AG modifies the topology of the circuit and the Jacobian matrix is no longer singular at the turning points. This is because the AG eliminates one of the state variables contributing to the singularity of the HB Jacobian matrix. Indeed, the voltage component $V_{n}$ at the AG frequency, at the node $n$ where this generator is connected, disappears as a variable, since its value is determined by the $\mathrm{AG}$, which is an independent voltage source: $V_{n}=A_{A G} e^{j 0}$. Therefore, the sweep of the AG-related values [in phase $(\alpha)$, amplitude $\left(A_{A G}\right)$ and frequency $\left(\omega_{A G}\right)$ ] that is used to calculate the collection of functions $Y_{A G}^{\alpha}\left(A_{A G}, \omega_{A G}\right)$ should not give rise to any convergence problems. In fact, during these sweeps the circuit is not optimized but behaves in a totally forced manner. The actual solution curve(s) are obtained at a later stage from the functions $Y_{A G}^{\alpha}\left(A_{A G}, \omega_{A G}\right)$, by means of the described contourintersection method.

A different situation is faced when optimizing/calculating of the auxiliary generator values in order to fulfill the nonperturbation condition $Y_{A G}=0$, as done in previous works [21][22],[29]. This constitutes an outer tier equation, to be fulfilled with the pure HB system as an inner tier. Then, at the turning points, one will have:

$$
\operatorname{det}[J Y]=\operatorname{det}\left[\begin{array}{ll}
\frac{\partial Y_{A G}^{r}}{\partial A_{A G}} & \frac{\partial Y_{A G}^{r}}{\partial \alpha} \\
\frac{\partial Y_{A G}^{i}}{\partial A_{A G}} & \frac{\partial Y_{A G}^{i}}{\partial \alpha}
\end{array}\right]=0
$$

which is easily derived from the conditions $\partial \alpha / \partial \omega_{i n}=\infty$ and $\partial A_{A G} / \partial \omega_{i n}=\infty$. As a result, when optimizing in order to fulfill $Y_{A G}=0$, it will be necessary to switch the analysis parameter to either $A_{A G}$ or $\alpha$.

In contrast with the previous case, the method based on the intersection of the contour plots $C_{r}^{\alpha}=\operatorname{Re}\left[Y_{A G \text {,int }}^{\alpha}\right]=0, C_{i}^{\alpha}=\operatorname{Im}\left[Y_{A G, \text { int }}^{\alpha}\right]=0$ does not exhibit any geometrical difficulties about the turning point. When passing through a turning point of the solution curve versus the parameter, the evolving intersection point simply changes its sense of variation with respect to this parameter, in agreement with the condition $\partial \omega_{A G, S} / \partial \alpha=0$. When varying $\alpha$ continuously, the two contour plots in (8), whose intersections provide the steady-state solutions, also evolve in a continuous manner. This is shown in Fig. 9, which presents the evolution of the two contours and their intersection points near $\mathrm{T}_{2}$ in Fig. 5(c). When passing through a turning point of the solution curve versus the parameter (such as $\mathrm{T}_{2}$ ), the evolving intersection point simply changes its sense of variation with respect to the parameter, in agreement with the condition $\partial \omega_{A G, s} / \partial \alpha=0$.

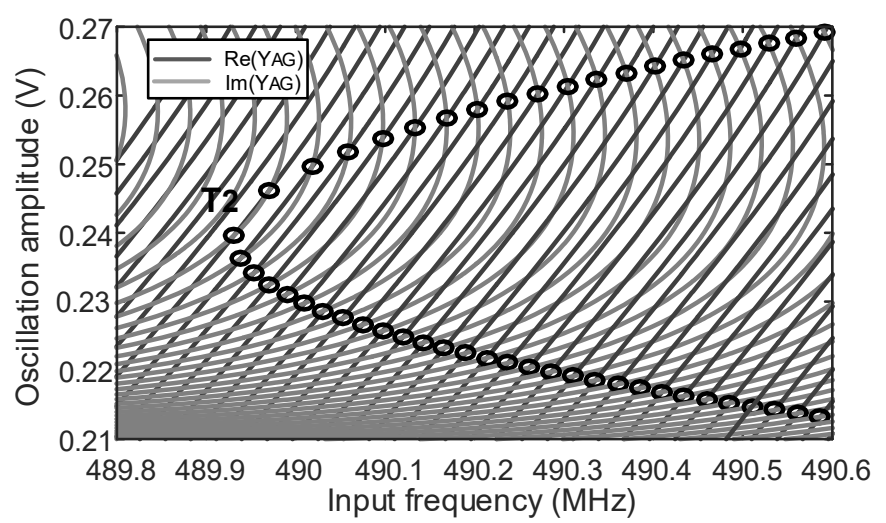

Fig. 9. Evolution of the contour plots near the turning point $T_{2}$, in (8) versus the input phase $\alpha$. The actual sub-synchronized solutions correspond to the intersections of these contour plots, represented with circles.

By setting the oscillator capacitors to $C_{1}{ }^{\prime}=2 \mathrm{pF}, C_{2}{ }^{\prime}=0.92$ $\mathrm{pF}$, and keeping the input frequency in the same interval considered in Fig. 7, one obtains the synchronized-solution curves in Fig. 10. Curve sections obtained through parameter switching (when convergence is achieved) are superimposed for validation. For $P_{i n}=16.45 \mathrm{dBm}$, one obtains a closed curve with a distorted "eight" shape, together with the usual lowamplitude curve, in which the self-oscillation is not excited. The eight-shaped is constituted by two sections, one corresponding to the subharmonic order $N=15$ and the other corresponding to $N=16$. This is gathered from the frequency range of each section and the inspection of output spectrum. In the upperfrequency section, the highest spectral line is $15 \omega_{i n}$ whereas in the lower-frequency section, the highest spectral line is $16 \omega_{i n}$. At $P_{\text {in }}=16.85 \mathrm{dBm}$, the former closed curve has split into two distinct curves. The one on the right side is closed and quite irregular, and the one on the left side is open as a result of the merging with the low amplitude curve (the one that coexisted with the closed one for the smaller $P_{\text {in }}$ values). The narrowing in the upper section of the closed curve, obtained for $P_{i n}=16.45$ $\mathrm{dBm}$, has evolved into a loop in the newly formed open curve. This quick evolution of the solution curves when varying the input power has been found to be a distinct feature of high-order subharmonic injection locking. Note that even when using an in-house HB simulator with powerful continuation methods, the isolated curve on the right, obtained for $P_{i n}=16.85 \mathrm{dBm}$, would have been missed. The new methodology succeeds in reaching this solution because it is not constrained by the local quality inherent to continuation methods. 


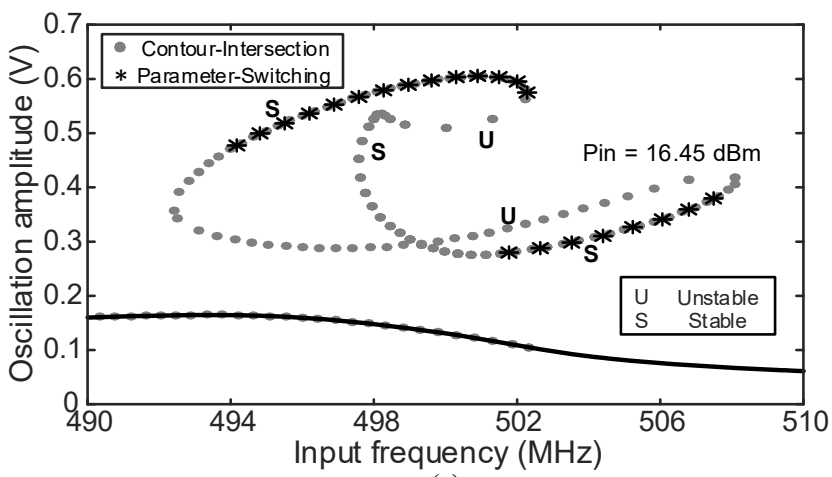

(a)

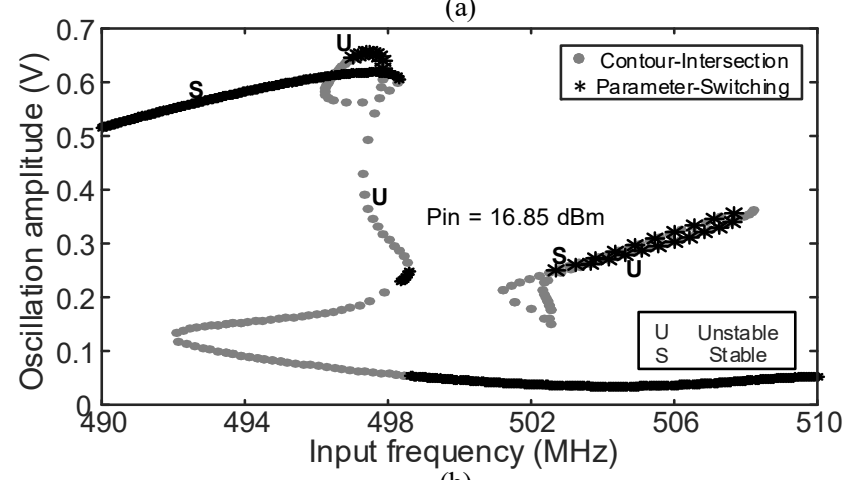

(b)

Fig. 10. Circuit in Fig. 3 at $8.05 \mathrm{GHz}$, with the oscillator capacitors $C_{1}{ }^{\prime}=2 \mathrm{pF}$, $C_{2}{ }^{\prime}=0.92 \mathrm{pF}$. Synchronized-solution curves versus $f_{\text {in }}$ for different $P_{\text {in }}$ values at $N=16$. Curve sections obtained through parameter switching (when convergence is achieved) are superimposed for validation. Stable and unstable sections resulting from the analysis described in Section IV.C are indicated. The free-running solution (not represented) is given by $V_{o}=1.33 \mathrm{~V}, f_{o}=503 \mathrm{MHz}$. (a) $P_{\text {in }}=16.45 \mathrm{dBm}$. (b) $P_{\text {in }}=16.85 \mathrm{dBm}$.

One must emphasize that it will not always be possible to trace the multi-valued solution curves through the previous technique [21]-[22],[31] based on AG parameter switching in commercial $\mathrm{HB}$, due to increasing error in the optimization goal $Y_{A G}$ and/or unexpected jumps between curve sections. Unlike previous methods [19]-[22],[31], in the new one the circuit is analyzed in a fully forced manner, without any additional oscillation condition, which significantly relaxes the analysis complexity. The HB software is only used to calculate the surfaces (5a) and (5b) which are interpolated at a later stage. Unlike methodologies based on continuation techniques, the new method is not local, in the sense that it enables a global exploration of all the coexistent solutions for given $\alpha$. This capability could be of interest even for in-house software with sophisticated continuation methods, since it is able to detect solutions in disconnected curves. The fact that the synchronization frequency interval is inherently limited in injection-locked circuits, and so are the usual amplitude variations in microwave circuits, will enable a moderate computational cost, further reduced by the data processing.

To summarize, the accuracy of the new method has been validated with AG-based parameter switching and fullyindependent envelope-transient simulations. The results are overlapped in the curve sections where these methods succeeded to converge. However, there are sections of the curves where the two mentioned methods were unable to provide any solutions.

\section{Stability analysis}

After obtaining the AG values that constitute valid solutions (since they fulfill $Y_{A G}=0$ ), one can supply these values to the $\mathrm{AG}$ in the $\mathrm{HB}$ simulator, which will directly force these solutions with no need of an optimization procedure. This way it will be possible to apply a complementary stability analysis [22], [29], enabling a distinction between physical and unphysical sections of the solution curves.

In general terms, the stability boundaries of injection-locked oscillators are determined by the generation of an invariant closed curve at a saddle node of the Poincaré map, occurring at turning point of the synchronized solution curve, or by a direct Hopf bifurcation [16]-[17],[22],[41]. Usually the turning-point desynchronization is obtained at the lower input power. In the two cases there is a transition from periodic to autonomous quasi-periodic regime. This transition is discontinuous in the case of the turning-point desynchronization (since the invariant closed curve is generated from the saddle-node manifolds) and continuous in the case of the Hopf bifurcation, at which unlocked oscillation amplitude tends to zero value. For higher input-power values one can also obtain turning points associated to hysteresis and jumps. Other forms of global bifurcations, such as the saddle connections in the Poincaré map also exist but are typically obtained in small parameter regions [16]-[17],[41].

The stability of the synchronized solution curves will be analyzed here through pole-zero identification [26]-[28]. This requires the calculation of a closed-loop transfer function at the perturbation frequency $\Omega$, incommensurable with $\omega_{i n}$. This function is obtained by introducing a small-signal current source $I_{s}$ at $\Omega$, in parallel at a sensitive circuit node, such as a device terminal. The closed-loop transfer function is given by the ratio between the voltage at the connection node $V_{s}$ and $I_{s}$ : $Z(\Omega)=V_{s} / I_{s}$. This complex function is fitted with a quotient of polynomials externally to the harmonic-balance software [26][28].

A problem specific to the high-order subharmonic injection locking is the selection of the identification frequency band, due to the significant difference between the oscillation frequency $\omega_{o}$ and the input frequency $\omega_{i n}$. In fact, the accuracy of the polezero increases when considering narrower identification bandwidths [28], in which the transfer function can be fitted with a smaller number of zeroes and poles. One should take into account that for a harmonic order tending to infinite, the poles of a periodic solution have been demonstrated to agree with the Floquet exponents $\lambda_{k}$ of this solution [27], [42]. These exponents are non-univocally related with the Floquet multipliers $m_{k}$ through $m_{k}=\exp \left[\left(\lambda_{k}+n j \omega_{i n}\right) T_{i n}\right]$, where $n$ is an integer number. Therefore, all the poles $\lambda_{k}+n j \omega_{i n}$ will be associated with a same Floquet multiplier $m_{k}$. As a result, there should be a "repetition" of the poles at multiples of $\omega_{i n}$. For illustration, a particular pair of complex-conjugate poles of the form: $\sigma \pm j \omega_{a}$ will be considered. Without loss of generality, it is assumed that $0<\omega_{a}<\omega_{\text {in }} / 2$. Then, a whole set of poles associated with the same pair of complex-conjugate multipliers 
$m_{k}=\exp \left[\left(\sigma \pm j \omega_{a}+n j \omega_{i n}\right) T_{i n}\right]$ will also exist, given by $\sigma \pm j\left(n \omega_{i n}-\right.$ $\left.\omega_{a}\right), \sigma \pm j\left(n \omega_{i n}+\omega_{a}\right)$.

In view of the above relationship between the poles and the Floquet multipliers, one should ideally obtain the same stability information when identifying $Z(\Omega)$ in any frequency band $\left[(n-1 / 2) \omega_{i n},(n+1 / 2) \omega_{i n}\right]$. In practice, some bands can be more sensitive than other, and one should expect a higher sensitivity in sub-bands around the original oscillation frequency. Nevertheless, in the oscillator considered here, the same stability information was obtained about $N \omega_{\text {in }}$ and in the lower sub-band $\left(0, \omega_{i n}\right)$.

As stated, the AG is used to force each solution (in the set obtained through the contour-plot intersections) in the commercial HB software. Then, the conversion matrix approach is applied for the calculation of the closed-loop transfer function $Z(\Omega)$, analyzed with pole-zero identification. This analysis is illustrated through its application to the most interesting case in Fig. 7, corresponding to the input amplitude $E_{i n}=2.95 \mathrm{~V}$. For convenience, the two solution curves obtained for this $E_{\text {in }}$ value have been retraced in Fig. 11(a).

The first solution considered corresponds to $N f_{\text {in }}=15 f_{\text {in }}=7.286 \mathrm{GHz}\left(f_{\text {in }}=485.7 \mathrm{MHz}\right)$, in the upper section of the main (open) curve. Fig. 11(b) shows the transfer function $Z(\Omega)$ evaluated in the perturbation-frequency interval $\Omega / 2 \pi=$ $f \in\left(15 f_{\text {in }}-f_{\text {in }} / 2,15 f_{\text {in }}+f_{\text {in }} / 2\right)$. As expected, the frequency response exhibits certain degree of symmetry about $15 f_{\text {in }}$. Due the fast frequency variations of $Z(\Omega)$, the identification must be carried out considering smaller frequency intervals [27]-[28]. One of the intervals is about $15 f_{\text {in }}$ and the other captures the most pronounced resonance on the left side. The identification of Fig. 11(b) about $15 f_{\text {in }}$ provides two pairs of complex-conjugate poles $\sigma_{1} \pm j\left(2 \pi 15 f_{i n} \pm 2 \pi \Delta f_{1}\right)$ at small offset frequency $\Delta f_{1}$ from $15 f_{i n}$, located on the left-hand side of the complex plane (LHS). The identification about the left resonance provides the complex conjugate poles $\sigma_{2} \pm j\left(2 \pi 15 f_{\text {in }} \pm 2 \pi \Delta f_{2}\right)$ at $\Delta f_{2}=162$ MHz located on the right-hand side (RHS). When reducing $f_{\text {in }}$ along the upper section of the main solution curve in Fig. 11(a), we obtain the pole evolution shown in Fig. 11(c). As the turning point $\mathrm{T}_{4}$ is approached, $\Delta f_{1}$ decreases. Just before $\mathrm{T}_{4}$, the poles $\sigma_{1} \pm j\left(2 \pi 15 f_{\text {in }} \pm 2 \pi \Delta f_{1}\right)$ merge and split into two independent pairs of poles $\sigma_{1}{ }^{\prime} \pm j\left(2 \pi 15 f_{\text {in }}\right)$ and $\sigma_{1}{ }^{\prime \prime} \pm j\left(2 \pi 15 f_{\text {in }}\right)$ (equivalent to two independent real poles) and, at $\mathrm{T}_{4}$, one of the pairs $\left[\sigma_{1} " \pm j\left(2 \pi 15 f_{i n}\right)\right]$ crosses to the RHS. Both the merging and crossing to the RHS occur in a very quick evolution. The pole crossing to the RHS is confirmed by the pole plot of Fig. 11(d), just after $\mathrm{T}_{4}$. The same pair of poles $\sigma_{1} " \pm j\left(2 \pi 15 f_{\text {in }}\right)$ crosses to the LHS after $T_{3}$ [Fig. 11(e)].
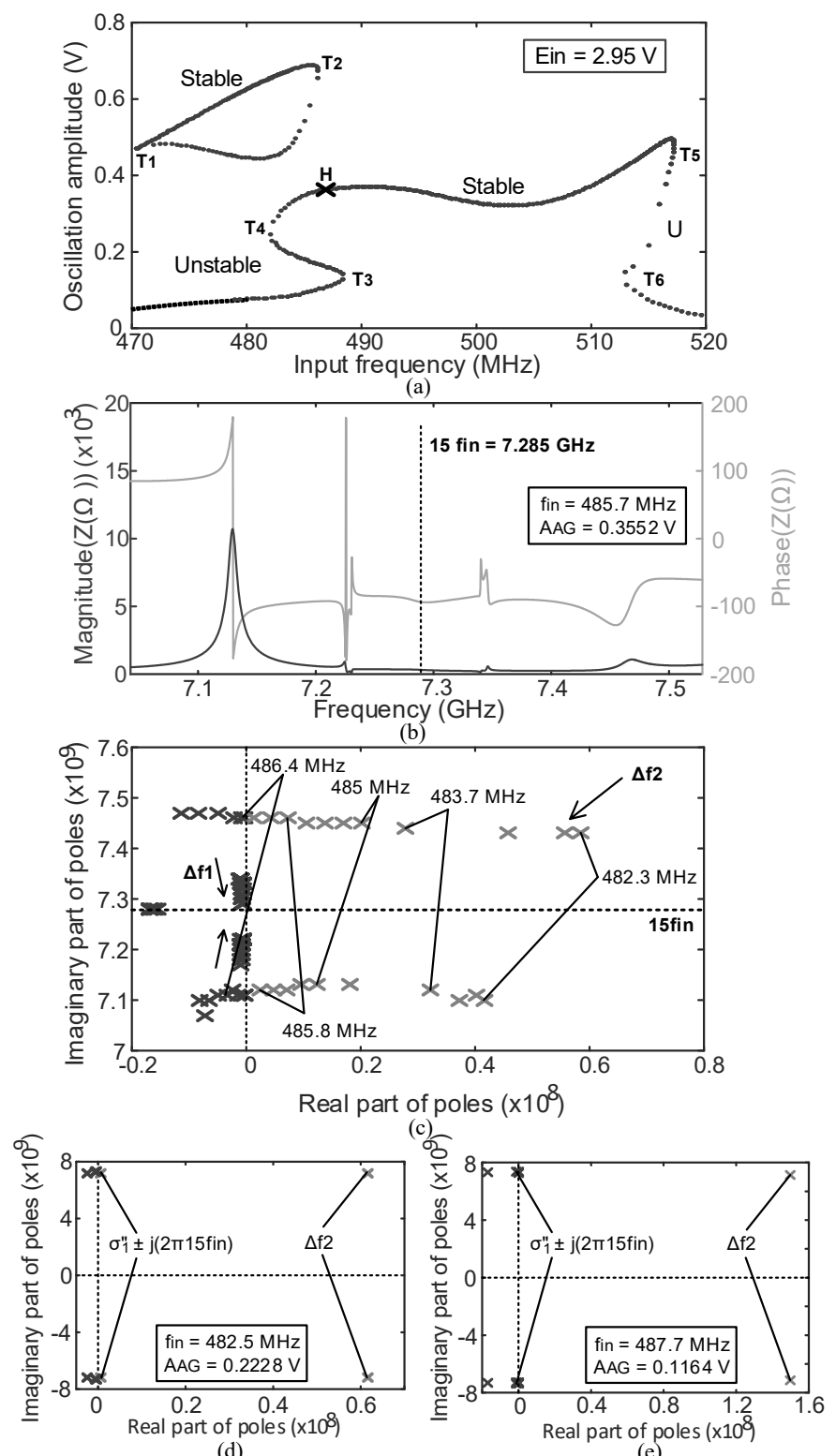

Fig. 11. Stability analysis of the circuit at $7.6 \mathrm{GHz}$ in Fig. 3. (a) Solution curve at $E_{\text {in }}=2.95 \mathrm{~V}$ and sub-synchronization order $N=15$. (b) Transfer function $Z(\Omega)$ calculated by connecting a current source $I_{s}$ in parallel at the drain terminal. (c) Evolution of poles when decreasing the input frequency $\mathrm{T}_{4}$. (d) Pole locus for $f_{\text {in }}=482.5 \mathrm{MHz}$, between $\mathrm{T}_{4}$ and $\mathrm{T}_{3}$. (e) Pole locus for $f_{\text {in }}=487.7$ $\mathrm{MHz}$, after $\mathrm{T}_{3}$.

Attention is now paid to the other dominant poles at $\sigma_{2} \pm j\left(2 \pi 15 f_{\text {in }} \pm 2 \pi \Delta f_{2}\right)$. The upper section of the main curve is stable from $\mathrm{H}$ to $\mathrm{T}_{5}$. However, when reducing $f_{i n}$, the poles at $\Delta f_{2}$ cross to the RHS at the direct Hopf bifurcation $\mathrm{H}$, obtained before reaching $\mathrm{T}_{4}$. This Hopf bifurcation leads to a quasiperiodic regime at $f_{\text {in }}$ and the unlocked oscillation frequency. The poles at $\Delta f_{2}$ remain in the RHS through the entire left section of the main curve, so the turning point $T_{4}$ has no physical effect. In fact, the pair of poles $\sigma_{1} " \pm j\left(2 \pi 15 f_{i n}\right)$ crosses again to the LHS at $\mathrm{T}_{3}$.

From the quasi-periodic regime that originates at $\mathrm{H}$, the circuit immediately evolves to a locked solution at $16 f_{i n}$, which is attained at the right turning point $\left(\mathrm{T}_{2}\right)$ of the closed curve on 
the left. This turning point is a synchronization point [29], in consistency with the fact that the closed curve is stable between the two turning points $T_{1}$ and $T_{2}$ and there is no coexistent stable section in the open curve in the same frequency interval. On the other hand, the right side of the main solution curve does not exhibit any Hopf bifurcations, so the upper section of the open curve remains stable up to $T_{5}$.

The results of the above stability analysis are consistent with the envelope-transient simulations, superimposed in Fig. 7. However, the envelope-transient analysis is only indicative [22], as it will fail if the solution involves frequencies not accounted for in the variable representation (as a Fourier series, with time-varying harmonic terms). Because the target of Fig. 7 is the comparison with independent analysis method, the information on the stable synchronization bands obtained for different $P_{\text {in }}$ is given in Table I. The results of the stability analysis through pole-zero identification are indicated in Fig. 10.

Table I First design. Synchronization bandwidths $N=15$

\begin{tabular}{cccc}
\hline$E_{\text {in }}$ & $f_{1}$ & $f_{2}$ & $B W$ \\
\hline $2.5 \mathrm{~V}$ & $7.517 \mathrm{GHz}$ & $7.674 \mathrm{GHz}$ & $157 \mathrm{MHz}$ \\
$2.65 \mathrm{~V}$ & $7.458 \mathrm{GHz}$ & $7.699 \mathrm{GHz}$ & $241 \mathrm{MHz}$ \\
$2.8 \mathrm{~V}$ & $7.425 \mathrm{GHz}$ & $7.728 \mathrm{GHz}$ & $303 \mathrm{MHz}$ \\
$2.95 \mathrm{~V}$ & $7.296 \mathrm{GHz}$ & $7.750 \mathrm{GHz}$ & $454 \mathrm{MHz}$ \\
\hline \hline
\end{tabular}

\section{EXPERIMENTAL RESULTS}

To validate the new analysis method with experimental results, it is necessary to take into account the package inductor of the varactor diodes, which would not be present in a MMIC realization. In fact, the package inductance has been found to be the most limiting effect in the performance of the NLTLdriven subharmonically injection-locked oscillator. Fig. 12(a) shows the measured output-power spectrum of the NLTL, when characterized isolated from the circuit, and driven with the input power $P_{\text {in }}=17 \mathrm{dBm}$. Seven significant harmonic components can be identified. Simulation results are also shown in Fig. 12(b). As stated, to achieve sub-synchronization an additional multiplication effect, due to the transistor device in the oscillatory regime, will be required. The two multiplication actions are not independent since, due to the mutual coupling between the NLTL and the transistor stage, the circuit operates as a single oscillator. In view of the degradation of the NLTL frequency-multiplication capabilities, we have redesigned the oscillator, so as to obtain an oscillation frequency about 4.3 $\mathrm{GHz}$. The input frequency has also been slightly reduced to achieve the sub-synchronization order to $N=11$, instead of $N=10$.

Fig. 13(a) shows a detailed schematic of the second design operating at $4.3 \mathrm{GHz}$, also based on the FET transistor NE3210S01. It has been built on Rogers 4003C substrate $\left(\varepsilon_{r}=3.38, H=30\right.$ mils), using TE connectivity 3640 series inductors and ATC 600S Series capacitors. The dc bias feeding networks have been implemented with radial stubs. The photograph is shown in Fig. 13(b).
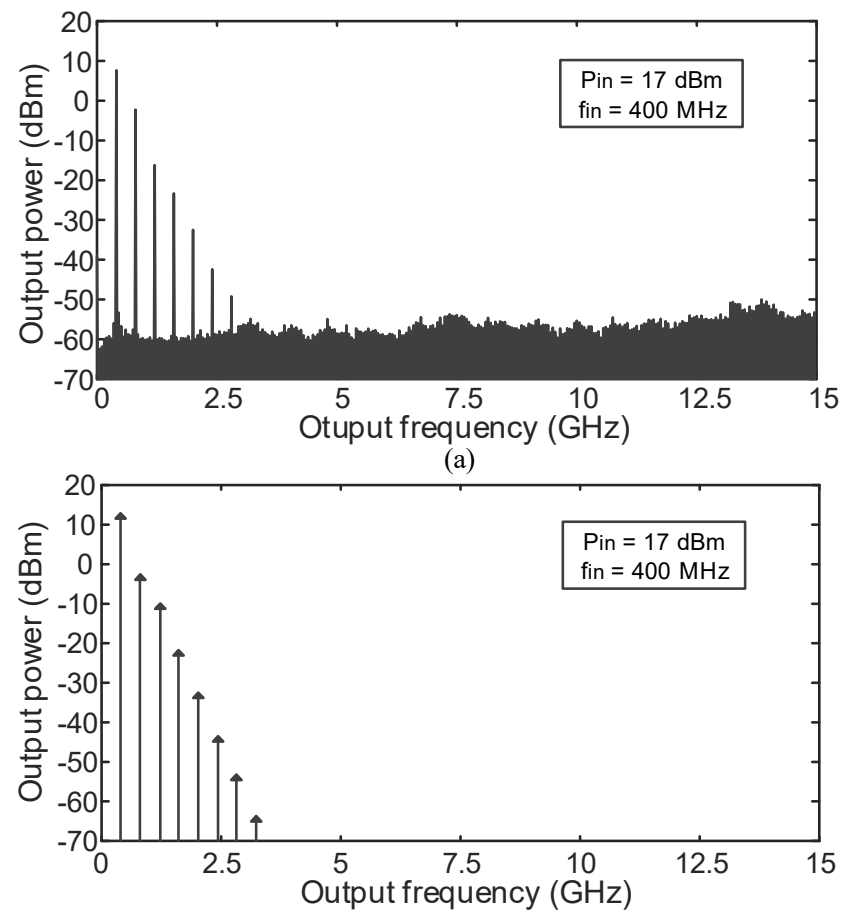

(b)

Fig. 12. Output spectrum of the NLTL. (a) Measured. (b) Simulated. The package inductor of the varactor diodes has been taken into account in the simulations.

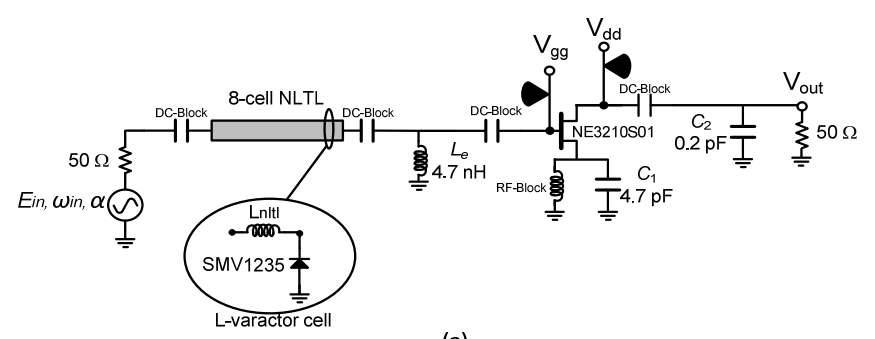

(a)

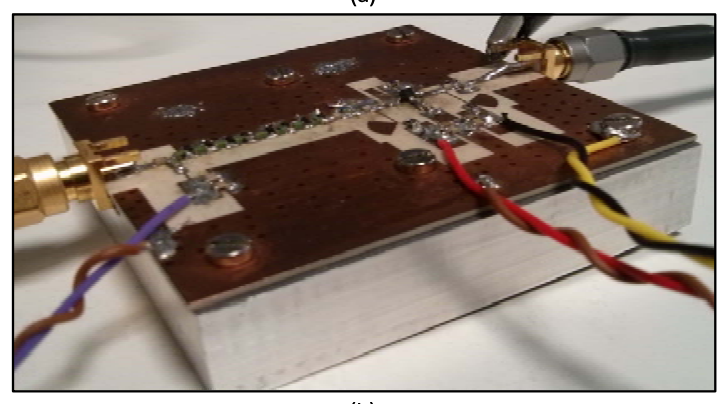

(b)

Fig. 13. Second design of the subharmonically injection-locked oscillator based on the transistor NE3210S01 and operating at $4.38 \mathrm{GHz}$. The results of the new analysis methodology are compared with experimental measurements. (a) Detailed circuit schematic. (b) Photograph of the Rogers prototype.

The synchronized solution curves, when considering the package inductor, are shown in Fig. 14(a). Each curve has been automatically obtained with the new contour-intersection method. The low pass filtering effects associated with the package inductor give rise to a reduction of the oscillation frequency. At $P_{\text {in }}=18.38 \mathrm{dBm}$ and $18.46 \mathrm{dBm}$, a closed curve and a disconnected open curve are obtained. One should note that the open curve obtained for $18.46 \mathrm{dBm}$ exhibits two turning points, which evidences the complexity of the solutions, even 
in the presence of the parasitic inductors. The measured synchronization bands for similar input power values are shown in Fig. 14(b). To match the synchronization band, it was necessary to use a lower drain-bias voltage $V_{\text {drain }}=0.75 \mathrm{~V}$ [43]. This is attributed to inaccuracies in the transistor model, as well as the big dispersion between device units, gathered from the cut-off voltage and saturated drain-current values in the datasheet [44]. When increasing $V_{\text {drain }}$, the synchronizedsolution curves shift to lower frequencies and the output power decreases. Fig. 14(c) shows the measured synchronization bands at $V_{\text {drain }}=1.7 \mathrm{~V}$. Measurements and simulations are in good qualitative agreement. Table II presents a summary of the measurement results for $V_{\text {drain }}=0.75 \mathrm{~V}$, in terms of synchronization band and output power at the middle frequency of the band $f_{\text {mid }}$. In comparison with the results of the first oscillator in Fig. 3, there is a significant reduction of the synchronization band, which is attributed to the degradation of the NLTL performance when using packaged inductors.

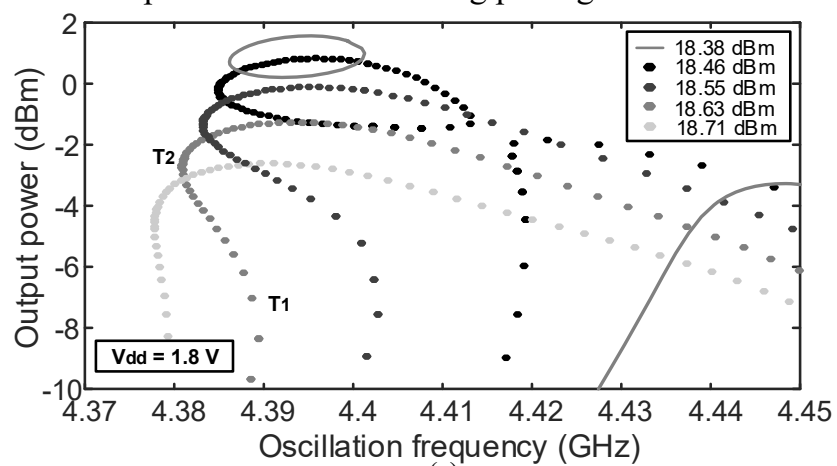

(a)

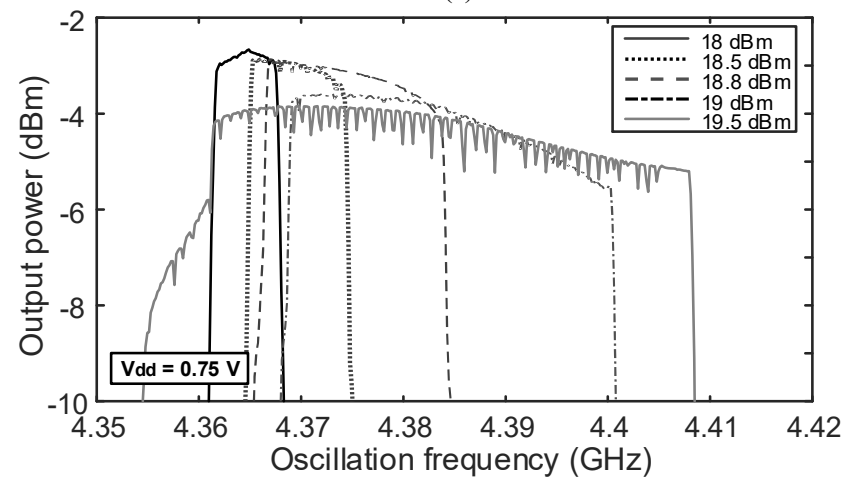

(b)

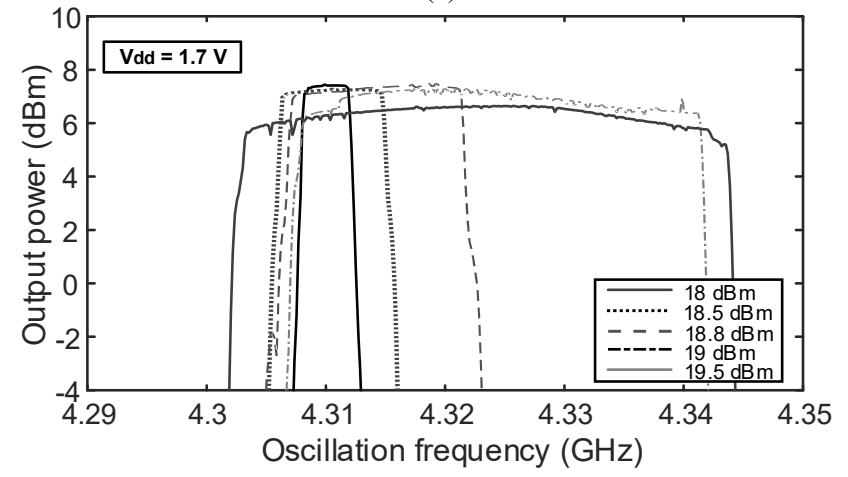

(c)

Fig. 14. Oscillator in Fig. 13, with complete varactor models, including the package inductor. (a) Synchronization curves obtained with the new analysis method for different $P_{\text {in }}$ values. (b) Experimental measurements for $V_{\text {drain }}=0.75$ V. (c) Experimental measurements for $V_{\text {drain }}=1.7 \mathrm{~V}$.

\begin{tabular}{ccccc}
\hline \hline \multicolumn{5}{c}{ Table II Measurement of } \\
\multicolumn{5}{c}{$\begin{array}{c}\text { synchronization bandwidths } \\
\mathbf{V}\end{array}$} \\
\hline \hline & $\boldsymbol{V}_{\text {drain }}=\mathbf{0 . 7 5}$ \\
$P_{\text {in }}$ & $f_{1}$ & $f_{2}$ & $B W$ & $P_{\text {out }}$ at $f_{\text {mid }}$ \\
\hline $18.5 \mathrm{dBm}$ & $4.365 \mathrm{GHz}$ & $4.374 \mathrm{GHz}$ & $9 \mathrm{MHz}$ & $-3 \mathrm{dBm}$ \\
$18.8 \mathrm{dBm}$ & $4.367 \mathrm{GHz}$ & $4.384 \mathrm{GHz}$ & $17 \mathrm{MHz}$ & $-3.1 \mathrm{dBm}$ \\
$19 \mathrm{dBm}$ & $4.369 \mathrm{GHz}$ & $4.4 \mathrm{GHz}$ & $31 \mathrm{MHz}$ & $-4 \mathrm{dBm}$ \\
$19.5 \mathrm{dBm}$ & $4.361 \mathrm{GHz}$ & $4.408 \mathrm{GHz}$ & $47 \mathrm{MHz}$ & $-4.1 \mathrm{dBm}$ \\
\hline \hline
\end{tabular}

Fig. 15 presents the measured pulsed waveform at the gate node, after the NLTL action, and at the circuit output for $V_{\text {drain }}=$ $0.75 \mathrm{~V}$, with a clear synchronized behavior, at the original. These waveforms have been obtained using a DSO90804A Digital Storage Oscilloscope. The pulse waveforms at the end of the NLTL were measured using the Agilent 1169A differential probes, which enable to test differential and singleended signals up to $12 \mathrm{GHz}$.

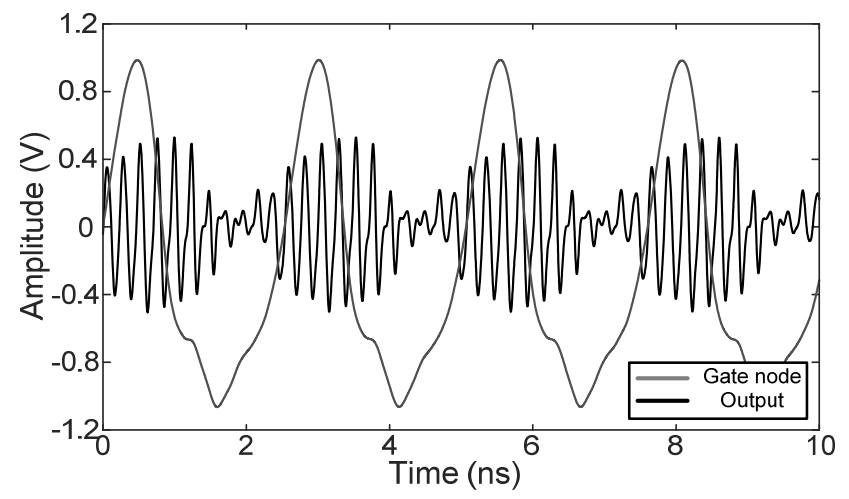

Fig. 15. Measured pulsed waveform at the gate node, after the NLTL action, and at the circuit output, evidencing the synchronized behavior.

Fig. 16 compares the simulated and experimental output spectrum of the subharmonically injection-locked oscillator. It has been measured with an Agilent PSA Series Spectrum Analyzer E4446A, connecting the output node of the circuit to the instrument through a cable of length $l=124 \mathrm{~cm}$. The noise floor in the measurement seems to be low, or lower than in Fig. 12(a), due to the adjustmet in the mixing level of the instrument. to avoid reaching its IF overloading (mixing level $=$ Input level - Attenuation $<-8 \mathrm{dBm}$ ). Note than in measured spectra of Fig. 12(a) and Fig. 16 (b)] the reference level is different and so the mixing level. The spectrum in Fig 16(b) has been measured setting a $15 \mathrm{GHz}$ frequency span, a reference level of $0 \mathrm{dBm}$ and a logarithmic scale of $10 \mathrm{~dB} / \mathrm{div}$. This output spectrum evidences the action of the active-device nonlinearity, since the NLTL alone is not able to provide any harmonic component near the oscillation frequency [Fig. 12(a)]. There are some discrepancies in the power values at the spectral lines, which are attributed to modeling inaccuracies. The spurious content about the main spectral line at $4.38 \mathrm{GHz}$ can be efficiently removed by using another injection-locked oscillator [5]-[6]. This oscillator [5]-[6] locks onto any harmonic of the pulsed oscillations included in its tuning range, through a harmonic selection by means of a tuning voltage. 

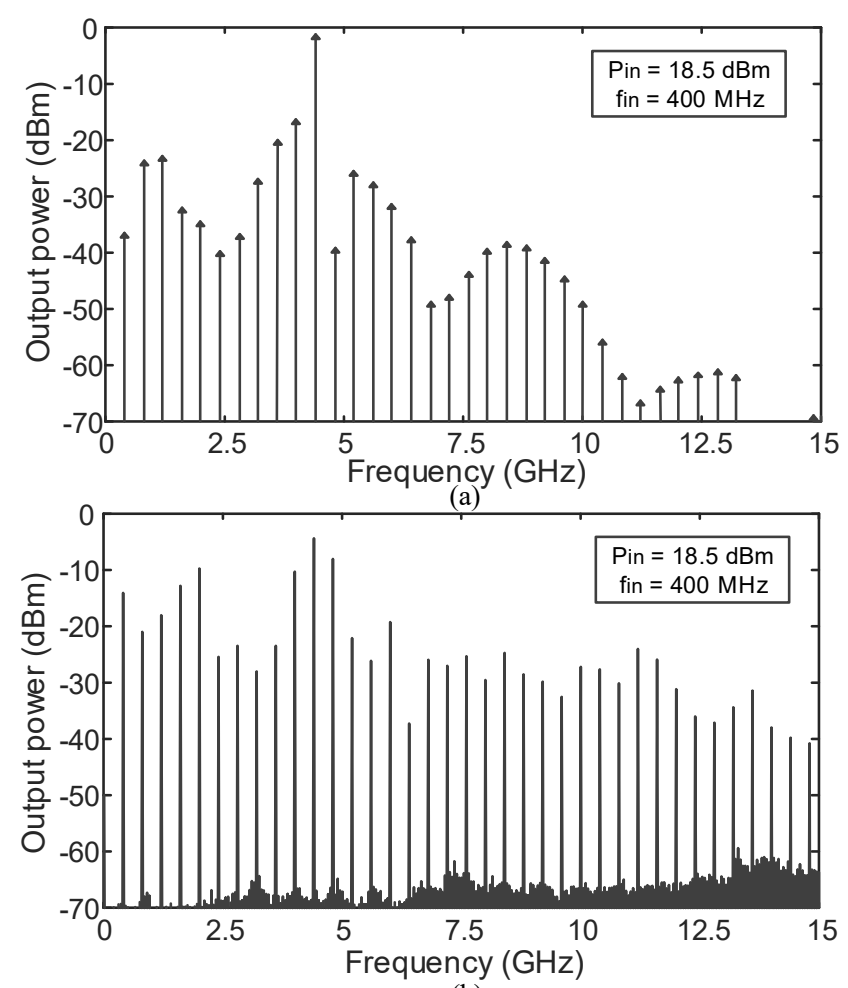

(b)

Fig. 16. Oscillator in Fig. 13. Output spectra with $P_{\text {in }}=18.5 \mathrm{dBm}$ and $f_{\text {in }}=400$ MHz. (a) Simulation. (b) Measurement.

The stability of the solution curves in Fig. 14(a) has been analyzed with pole-zero identification [26]-[28]. The analysis is illustrated through its application to the left section of the curve obtained for $P_{i n}=18.63 \mathrm{dBm}$. Fig. 17(a) shows the evolution of the dominant poles when taking the output power $P_{\text {out }}$ as the parameter. The analysis departs from $P_{\text {out }}=-1.31$ $\mathrm{dBm}$. At this power value there is a dominant pair of complexconjugate poles at $11 f_{\text {in }}+\Delta f$, located on the LHS. As $P_{\text {out }}$ decreases, $\Delta f$ becomes smaller and reaches zero at $P_{\text {out }}$ $=-2.4 \mathrm{dBm}$, where the original pair of incommensurable complex-conjugate poles splits into two independent pairs of poles at $\sigma_{1}{ }^{\prime} \pm j\left(2 \pi 11 f_{\text {in }}\right)$ and $\sigma_{1}{ }^{\prime \prime} \pm j\left(2 \pi 11 f_{\text {in }}\right.$ ) (equivalent to two independent real poles). At $\mathrm{T}_{2}$ [Fig. 14(a)], obtained for $P_{\text {out }}$ $=-2.5 \mathrm{dBm}$, one of the pairs $\left[\sigma_{1}{ }^{\prime} \pm j\left(2 \pi 15 f_{\text {in }}\right)\right]$ crosses to the RHS. When further reducing $P_{\text {out }}$, the second pair $\sigma_{1} " \pm j\left(2 \pi 11 f_{\text {in }}\right)$ crosses to the RHS at $P_{\text {out }}=-2.70 \mathrm{dBm}$, corresponding to the turning point $\mathrm{T}_{1}$ [Fig. 14(a)]. The right section of the curve, for frequencies higher than the one corresponding to $P_{\text {out }}=-1.31 \mathrm{dBm}$ was found to be stable in simulation, unlike what is observed in the measurements.

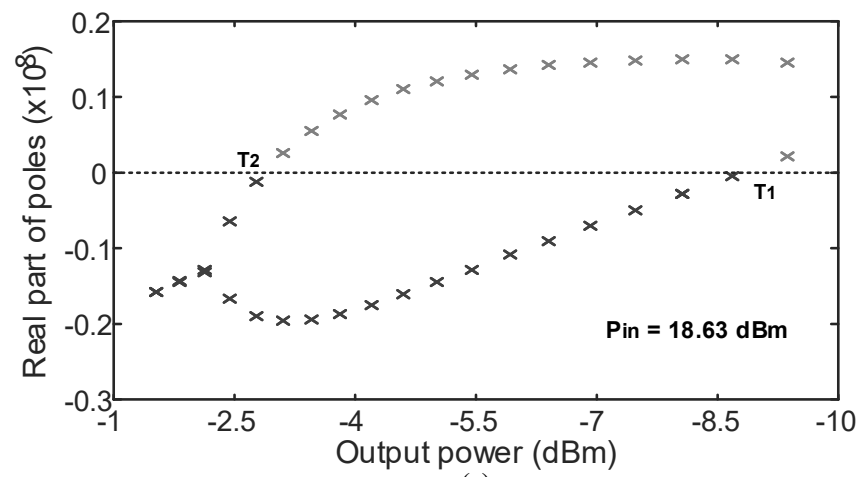

(a)

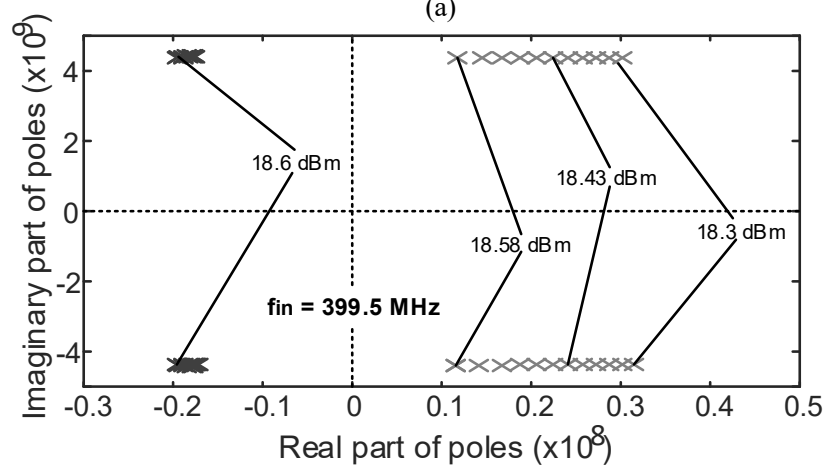

(b)

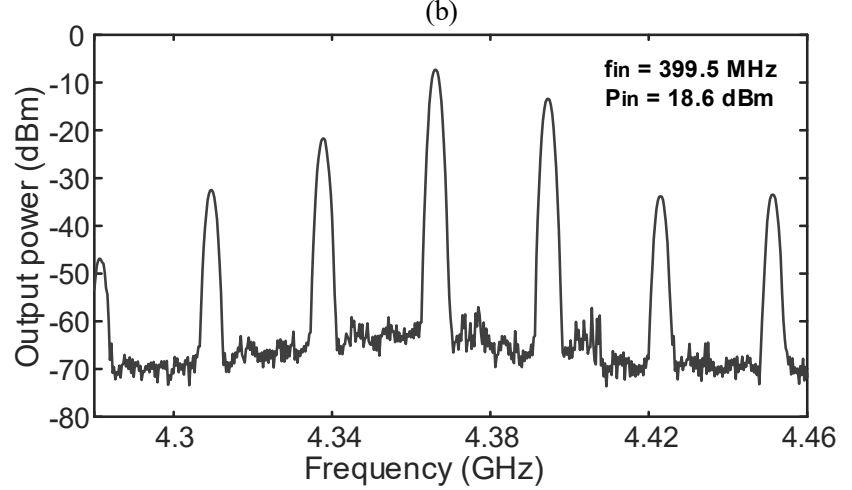

(c)

Fig. 17. Oscillator in Fig. 13. (a) Evolution of poles through $T_{2}$ and $T_{1}$ of the synchronization curve at $\mathrm{P}_{\text {in }}=18.63 \mathrm{dBm}$. (b) Evolution of poles at $f_{\text {in }}=399.5$ $\mathrm{MHz}$ when increasing the input power. (c) Measured spectrum at $11 f_{\text {in }}$ with $f_{\text {in }}$ $=399.5 \mathrm{MHz}$ before the extinction of the unlocked oscillation.

In the experiment, the circuit becomes unlocked at $15 f_{\text {in }}=4.374 \mathrm{GHz}$, where an incommensurable oscillation grows from zero amplitude [29], corresponding to a direct Hopf bifurcation. This Hopf bifurcation is not obtained in simulation for $P_{\text {in }}=18.63 \mathrm{dBm}$, but for lower input power. To demonstrate this, we have considered a constant input frequency $f_{\text {in }}=399.5$ $\mathrm{MHz}$ and increased $P_{\text {in }}$ from zero. Thus, the circuit departs from free-running conditions and one obtains a pair of complexconjugate poles at the oscillation frequency [Fig. 17(b)]. When increasing $P_{i n}$, the poles shift to the left and cross the imaginary axis at about $P_{\text {in }}=18.59 \mathrm{dBm}$, in a very quick evolution. Performing the same $P_{i n}$ variations experimentally, at $f_{\text {in }}=399.5 \mathrm{MHz}$, the circuit stabilizes at $P_{\text {in }}=18.7 \mathrm{dBm}$, higher than the result obtained in simulation. Fig. 17(c) shows the measured spectrum just before the extinction of the unlocked oscillation. Since there is significant spacing between the spectral lines, the oscillation extinction is due to an inverse Hopf bifurcation, in agreement with the analysis results. In our 
simulations of Fig. 14(a), the input power has surpassed the one required for the stabilization of the periodic solution.

The phase noise has been measured with the Direct Spectrum Technique, using the phase noise measurement personality in an Agilent PSA Spectrum Analyzer E4446A (option 226). The circuit was connected into the spectrum analyzer tuned to the carrier frequency, directly measuring the power spectral density of the oscillator in terms of single sideband phase noise $L(f)$ in $\mathrm{dBc} / \mathrm{Hz}$ versus $\log$ offset frequency. Fig. 18 compares the measured phase noise spectra of the input generator (HP 8648C) and the subharmonically injection-locked oscillator. The locked oscillator basically follows the phase-noise of the input source (increased in $20 \log 11 \mathrm{~dB}$ ). The phase-noise spectrum is also compared with the one obtained with the original implementation of the oscillator, without an NLTL, terminated in a grounded inductor at the gate port, of value $L=$ $0.85 \mathrm{nH}$. Despite the presence of a high number of varactor diodes in the NLTL driven oscillator, its phase noise is rather low in the whole interval of offset frequencies from the oscillator carrier. It is only higher than the one corresponding to the original free-running oscillator (without the NLTL) about $10 \mathrm{MHz}$ offset from the carrier. This is attributed to the relatively high noise floor of the input generator, since the expected difference $20 \log N$ is approximately maintained even at this high frequency offset.

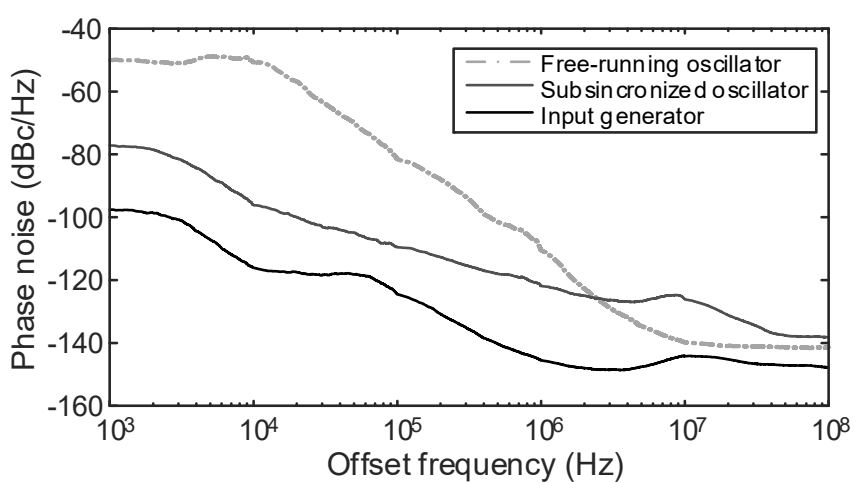

Fig. 18. Oscillator in Fig. 13. Measured phase noise spectra of the original freerunning oscillator (without NLTL), the input generator (HP 8648C) and the NLTL-driven subharmonically injection-locked oscillator.

\section{CONCLUSION}

A new methodology for the analysis of high order subharmonically injection-locked oscillators has been presented. Unlike previous methodologies, it is not local and therefore it is able to provide disconnected sections of solution curves and even disconnected curves. The method is based on a straightforward calculation of a collection of admittance functions versus the input-generator phase, using an auxiliary generator in standard harmonic balance. No continuation methods are required, except the ordinary continuation employed in any harmonic balance sweep. All the solutions coexisting for a given value of the input-generator phase are directly obtained from the intersections of two contours, respectively defined by the intersections of the surfaces associated with the real and imaginary parts of the admittancefunction with the zero-value planes. This way it is possible to obtain very distant solutions, belonging to different curve sections and different curves. It is to note that the method is fully extensive to the analysis of frequency dividers, by calculating the surfaces in terms of the auxiliary generator phase. The new methodology is compatible with any harmonic balance software, including those in which no oscillator analysis method is available. To demonstrate the capabilities of the method, a subharmonic injection locked oscillator, using an NLTL for a first frequency-multiplication stage, has been analyzed in depth. The results have been validated with the previous AG parameter-switching method, with envelopetransient simulations and with measurements.

\section{ACKNOWLEDGMENT}

The authors would like to thank J. de Cos for his valuable help in the realization of this work and Dr. J.A. García from University of Cantabria for sharing his in-depth knowledge on transistor modelling with us.

\section{REFERENCES}

[1] U. L. Rohde, A. K. Poddar and G. Bock, The Design of Modern Microwave Oscillators for Wireless Applications, Wiley, Hoboken, NJ, 2005.

[2] F. X. Kaertner, "Determination of the correlation spectrum of oscillators with low noise," IEEE Trans. Microw. Theory Techn., vol. 37, no.1, pp. 90-101, Jan., 1989.

[3] A. Demir. "Phase noise in oscillators: DAEs and colored noise sources," IEEE/ACM Int. Comput.-Aided Design Conf.,, 1998, pp. 170-177.

[4] A. Hajimiri and T. H. Lee, "A general theory of phase noise in electrical oscillators," IEEE J. Solid-State Circuits, vol. 33, no. 2, pp. 179-194, Feb., 1998.

[5] C. Jany, A. Siligaris, P. Ferrari and P. Vincent, "A novel programmable harmonic selection technique based on the pseudo-locking of an oscillator by periodically repeated oscillations train," 44th European Microwave Conference (EuMC), Rome, Italy, 2014, pp. 1020-1023.

[6] C. Jany, A. Siligaris, J. L. Gonzalez-Jimenez, P. Vincent and P. Ferrari, "A Programmable Frequency Multiplier-by-29 Architecture for Millimeter Wave Applications," IEEE J. Solid-State Circuits, vol. 50, no. 7, pp. 16691679, Jul., 2015.

[7] E. Fernández, M. Pontón, A. Suárez and F. Ramirez, "Subharmonically injection-locked oscillator using a nonlinear transmission line," 9th European Microwave. Integrated Circuit Conference (EuMIC), Rome, Italy, 2014, pp. 112-115.

[8] F. Martín, X. Oriols, J. A. Gil, and J. García-García, "Optimization of nonlinear transmission lines for harmonic generation," Int. J. Infrared Millimeter Waves, vol. 23, no. 1, pp. 95-103, Jan., 2002.

[9] M. J. W. Rodwell, M. Kamegawa, R. Yu, M. Case, E. Carman and K. S. Giboney, "GaAs nonlinear transmission lines for picosecond pulse generation and millimeter-wave sampling," IEEE Trans. Microw. Theory Techn, vol. 39, no. 7, pp. 1194-1204, Jul., 1991.

[10]E. Afshari and A. Hajimiri, "Nonlinear transmission lines for pulse shaping in silicon," IEEE J. Solid-State Circuits, vol. 40, no. 3, pp. 744-752, Mar., 2005.

[11]M. Remoissenet, Waves Called Solitons: Concepts and Experiments, New York: Springer, 1999.

[12] X. Zhang, X. Zhou, and A. S. Daryoush, "A theoretical and experimental study of the noise behavior of subharmonically injection locked local oscillators," IEEE Trans. Microw. Theory Techn., vol. 40, no. 5, pp. 895902, May 1992.

[13]X. Zhou and A. S. Daryoush, "Efficient self-oscillating mixer for communications," IEEE Trans. Microw. Theory Techn., vol. 42, no. 10, pp. 1858-1862, Oct., 1994.

[14]X. Zhang, X. Zhou, B. Aliener and A. S. Daryoush, "A study of subharmonic injection locking for local oscillators," IEEE Microw. Guided Wave Lett., vol. 2, no. 3, pp. 97-99, Mar., 1992.

[15] E. Fernández, F. Ramírez, A. Suárez and S. Sancho, "Stability and phasenoise analysis of pulsed injection-locked oscillators," IEEE Trans. Microw. Theory Techn., vol. 61, no. 1, pp. 482-491, Jan., 2013.

[16] T. S. Parker and L.O. Chua, Practical Numerical Algorithms for Chaotic Systems, Springer-Verlag, Berlin, 1989. 
[17] J. Guckenheimer and P. Holmes, Nonlinear Oscillations, Dynamical Systems and Bifurcations of Vector Fields. New York: Springer-Verlag, 1990.

[18] S. Wiggins, Introduction to Applied Nonlinear Dynamical Systems and Chaos. New York: Springer-Verlag, 1990.

[19] R. Quéré, E. Ngoya, M. Camiade, A. Suarez, M. Hessane and J. Obregon, "Large signal design of broadband monolithic microwave frequency dividers and phase-locked oscillators," IEEE Trans. Microw. Theory Techn., vol. 41, no. 11, pp. 1928-1938, Nov., 1993.

[20] V. Rizzoli and A. Neri, "State of the art and present trends in nonlinear microwave CAD techniques," IEEE Trans. Microw. Theory Techn., vol. 36, no. 2, pp. 343-356, Feb., 1988.

[21]A. Suárez and R. Quéré, Stability Analysis of Nonlinear Microwave Circuits. Boston, MA: Artech House, 2003.

[22]A. Suárez, Analysis and Design of Autonomous Microwave Circuits. Hoboken, NJ: Wiley IEEE Pres, 2009.

[23] L. Chua and A. Ushida, "A switching parameter algorithm for finding multitple solutions of nonlinear resistive circuits," Int. J. Circ. Theor. Appl., vol. 4, no. 3, pp. 215-239, Jul. 1976.

[24] R. Courant, F. John, Introduction to Calculus and Analysis, Volume II., Springer-Verlag, 1989.

[25] R.C. Melville, L. Trajkovic, S.C. Fang, L.T. Watson, "Artificial Parameter Homotopy Methods for the DC Operating Point Problem", IEEE Trans. Comput.-Aided Des. Integr. Circuits Syst, vol. 12, no. 6, pp. 861-877, Jun., 1993.

[26] J. Jugo, J. Portilla, A. Anakabe, A. Suárez, and J. M. Collantes, "Closedloop stability analysis of microwave amplifiers," IEEE Electron. Lett., vol. 37, no. 4, pp. 226-228, Mar., 2001.

[27] J. M. Collantes, I. Lizarraga, A. Anakabe, and J. Jugo, "Stability verification of microwave circuits through Floquet multiplier analysis," Proc. IEEE Asia-Pacific Conf. Circuits Syst., Tainan, Taiwan, 2004, pp. 997-100.

[28] N. Ayllon, J. M. Collantes, A. Anakabe, I. Lizarraga, S. Soubercaze-Pun and S. Forestier, "Systematic approach to the stabilization of multitransistor circuits," IEEE Trans. Microw. Theory Techn., vol. 59, no. 8, pp. 2073-2082, Aug., 2011.

[29] A. Suarez, J. Morales and R. Quere, "Synchronization analysis of autonomous microwave circuits using new global-stability analysis tools," IEEE Trans. Circuits and Syst.I, vol. 46, no. 5, pp. 494-504, May 1998.

[30] A. Suárez and J. M. Collantes, "A new technique for chaos prediction in RF circuit design using harmonic-balance commercial simulators," IEEE Trans. Circuits and Syst.I, Fundam. Theory and App., vol. 46, no. 11, pp. 1413-1415, Nov., 1999.

[31] F. Ramirez, M. E. de Cos and A. Suárez, "Nonlinear analysis tools for the optimized design of harmonic-injection dividers," IEEE Trans. Microw. Theory Techn., vol. 51, no. 6, pp. 1752-1762, Jun., 2003.

[32] B. Razavi, "A study of injection locking and pulling in oscillators," IEEE J. Solid-State Circuits, vol. 39, no. 9, pp. 1415-1424, Sept., 2004.

[33] J. de Cos and A. Suárez, "Efficient Simulation of Solution Curves and Bifurcation Loci in Injection-Locked Oscillators," IEEE Trans. Microw. Theory Techn., vol. 63, no. 1, pp. 181-197, Jan., 2015.

[34] E. Ngoya and R. Larcheveque, "Envelop transient analysis: a new method for the transient and steady state analysis of microwave communication circuits and systems," IEEE MTT-S Int. Microwave Symp. Dig., San Francisco, CA, USA, 1996, vol.3, pp. 1365-1368.

[35] N. B. Carvalho, J. C. Pedro, W. Jang, and M. B. Steer, "Nonlinear RF circuits and system simulation when driven by several modulated signals", IEEE Trans. Trans. Microw. Theory Techn., vol. 54, no. 2, pp. 572-579, Feb., 2006.

[36] M. E. de Cos, A. Suarez and S. Sancho, "Envelope transient analysis of self-oscillating mixers," IEEE Trans. Microw. Theory Techn., vol. 52, no. 4, pp. 1090-1100, Apr., 2004.

[37] L. Gustafsson, K. I. Lundstrom and G. H. B. Hansson, "Basic Properties of Subharmonic Injection Locking," IEEE Trans. Trans. Microw. Theory Techn.,, vol. 21, no. 1, pp. 28-34, Jan 1973.

[38] M. Fernández, S. Ver Hoeye, C. Vázquez, G. Hotopan, R. Camblor and F. Las Heras, "Analysis of the locking range of rationally synchronized oscillators with high reference power", IEEE Trans. Trans. Microw. Theory Techn., vol. 60, no. 8, pp. 2494-2504, Jun., 2012.

[39] Advanced Design System (ADS2015), Keysight Technologies, Inc., 2015.

[40] MATLAB (R2015a), The Mathworks, Inc., Massachusetts, 2015.

[41]H. Kawakami, "Bifurcation of periodic responses in forced dynamic nonlinear circuits: Computation of bifurcation values of the system parameters," IEEE Trans. Circuits and Syst., vol. 31, no. 3, pp. 248-260, Mar., 1984.
[42] F. Bonani and M. Gilli, "Analysis of stability and bifurcations of limit cycles in Chua's circuit through the harmonic-balance approach," IEEE Trans. Circuits and Syst.I, vol. 46, no. 8, pp. 881-890, Aug., 1999.

[43] J.A. Garcia, E. Malaver, L. Cabria, C. Gómez, A. Mediavilla and A. Tazón, "Device-level intermodulation distortion control on III-V FET's", Proc. 11th GaAs Symp., Munich, Germany, Oct., 2003, pp. 77-79.

[44] California Eastern Laboratories (2004, Jan. 01), NEC's super low noise HJ FET. Available: http://www.cel.com/pdf/datasheets/ne3210s1.pdf

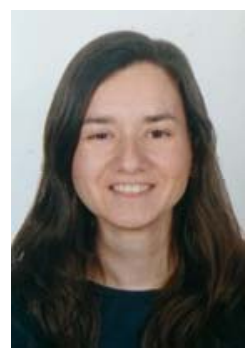

Silvia Hernández was born in the Canary Islands, Spain. She received her M.S. degree in Telecommunication Engineering from the University of Las Palmas de Gran Canaria (ULPGC), Canary Islands, Spain, in 2015. The same year, she entered the Institute for the Technological Development and Innovation in Communications (IDeTIC) at ULPGC as a research assistant. In 2016, she joined the Communications Engineering Department, University of Cantabria, where she is currently working towards her Ph.D. degree.

Her research interests include stability, phase-noise analysis and the study of new methods for the analysis and design of nonlinear microwave circuits.

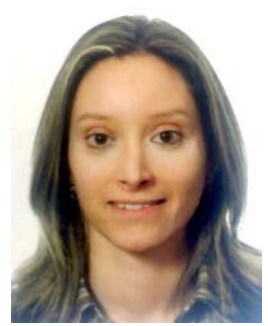

Mabel Pontón (S'08-M'11) was born in Santander, Spain. She received the bachelor's degree in telecommunication engineering, master's degree in information technologies and wireless communications systems, and Ph.D. degree from the University of Cantabria, Santander, in 2004, 2008, and 2010, respectively.

In 2006, she joined the Communications Engineering Department, University of Cantabria. From 2011 to 2013, she was with the Group of Electronic Design and Applications, Georgia Institute of Technology, Atlanta, GA, USA, as a Post-Doctoral Research Fellow.

Her current research interests include the nonlinear analysis and simulation of radiofrequency and microwave circuits, with an emphasis on phase-noise, stability, and bifurcation analysis of complex oscillator topologies.

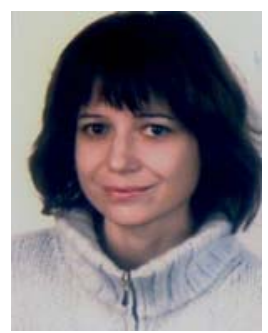

Almudena Suárez (M'96-SM'01-F'12) was born in Santander, Spain. She received the degree in electronic physics and the Ph.D. degree from the University of Cantabria, Santander, Spain, in 1987 and 1992, respectively, and the Ph.D. degree in electronics from the University of Limoges, France, in 1993. At present, she is a Full Professor at the University of Cantabria, and a member of its Communications Engineering Department. She has authored the book Analysis and design of autonomous microwave circuits for the publisher IEEE-Wiley and co-authored the book Stability analysis of microwave circuits for the publisher Artech-House. She belongs to the technical committees of the IEEE International Microwave Symposium and European Microwave Conference. She was an IEEE Distinguished Microwave Lecturer for the period 2006-2008. She is an associate editor of the IEEE Microwave Magazine. She is a member of the Board of Directors of EuMA and the Editor in Chief of International Journal of Microwave and Wireless Technologies from Cambridge University Press. 